\title{
Impaired phosphocreatine metabolism in white adipocytes promotes inflammation
}

\author{
Salwan Maqdasy $\mathbb{1}^{1,2,3,14}$, Simon Lecoutre ${ }^{1,14}$, Gianluca Renzi', Scott Frendo-Cumbo ${ }^{1}$, \\ David Rizo-Roca4, Thomas Moritz ${ }^{5,6}$, Marta Juvany ${ }^{5}$, Ondrej Hodek ${ }^{5}$, Hui Gao ${ }^{7}$, Morgane Couchet', \\ Michael Witting ${ }^{8,9,10}$, Alastair Kerr ${ }^{1}{ }^{1}$, Martin O. Bergo"1, Robin P. Choudhury ${ }^{12}$, Myriam Aouadi ${ }^{1}$, \\ Juleen R. Zierath $\mathbb{1}^{4,13}$, Anna Krook ${ }^{13}$, Niklas Mejhert $\mathbb{B}^{1,15} \bowtie$ and Mikael Rydén $\mathbb{1}^{1,15} \bowtie$
}

The mechanisms promoting disturbed white adipocyte function in obesity remain largely unclear. Herein, we integrate white adipose tissue (WAT) metabolomic and transcriptomic data from clinical cohorts and find that the WAT phosphocreatine/ creatine ratio is increased and creatine kinase-B expression and activity is decreased in the obese state. In human in vitro and murine in vivo models, we demonstrate that decreased phosphocreatine metabolism in white adipocytes alters adenosine monophosphate-activated protein kinase activity via effects on adenosine triphosphate/adenosine diphosphate levels, independently of WAT beigeing. This disturbance promotes a pro-inflammatory profile characterized, in part, by increased chemokine (C-C motif) ligand 2 (CCL2) production. These data suggest that the phosphocreatine/creatine system links cellular energy shuttling with pro-inflammatory responses in human and murine white adipocytes. Our findings provide unexpected perspectives on the mechanisms driving WAT inflammation in obesity and may present avenues to target adipocyte dysfunction.

O besity is characterized by excess white adipose tissue (WAT) mass and is linked to complications such as insulin resistance, type 2 diabetes, cardiovascular disease and many common cancers ${ }^{1,2}$. At the white adipocyte level, obesity perturbs multiple processes, including gene transcription and metabolic pathways and promotes a pro-inflammatory phenotype, alterations which together link obesity to many of its sequela $e^{3-6}$. However, the mediators driving these changes in fat cells remain unclear.

Studies in other tissues have shown that polar metabolites exert multiple effects on cell function, ranging from epigenetic changes, receptor binding and activation, to altered bioenergetic states ${ }^{7-10}$. In addition, studies in immune cells demonstrate that alterations in intracellular metabolism are tightly coupled to inflammatory status $^{11,12}$. Collectively, this demonstrates that metabolites can coordinate multiple cellular processes and that disturbances in their levels induce distinct changes in tissue function. Characterization of WAT metabolites in the obese and insulin-resistant states can, therefore, provide insights into the mechanisms that regulate tissue dysfunction $^{13}$. Despite this, the metabolic changes that characterize human obese WAT and how these impact on white adipocyte function and clinical phenotypes are unclear.

To identify pathways disturbed in conditions of excess fat mass, we performed unbiased metabolomic and transcriptomic analyses of human WAT and identified that phosphocreatine/creatine metabolism is perturbed in obesity. In white adipocytes, alterations in this energy shuttling system result in changes in adenosine triphosphate (ATP)/adenosine diphosphate (ADP) levels, which attenuate adenosine monophosphate-activated protein kinase (AMPK) activity, thereby promoting a pro-inflammatory profile. Together with recent studies in beige/brown adipocytes ${ }^{14-17}$ and macrophages ${ }^{18}$, our findings highlight the unique cell type-selective role of the phosphocreatine/creatine pathway and its importance in the regulation of obesity-accelerated inflammation in WAT.

\section{Results}

Obesity perturbs phosphocreatine metabolism in human WAT. To identify polar metabolites altered by excess fat mass, we performed an untargeted metabolomic analysis of subcutaneous abdominal WAT obtained from adult women with $(n=13)$ or without $(n=13)$ obesity (cohort 1 , described in ref. ${ }^{19}$ and Supplementary Table 1, Trial registration no. NCT01727245). Among all detected metabolites $(n=310)$, phosphocreatine displayed the highest fold-enrichment in the obese versus the non-obese state (Fig. 1a). Phosphocreatine and ADP are generated from creatine and ATP via specific creatine kinases (CK-MTs, CK-B and CK-M, forward reaction) where CK-B/CK-M can also catalyse the dephosphorylation back to creatine and ATP (reverse reaction) ${ }^{16}$. Cellular creatine can either be taken up directly via the plasma membrane transporter SLC6A8, or be synthesized de novo from the precursor guanidinoacetate, which in turn can be taken up by SLC6A6 or synthesized

'Department of Medicine (H7), Karolinska Institutet, Karolinska University Hospital Huddinge, Huddinge, Sweden. ${ }^{2} \mathrm{CHU}$ Clermont-Ferrand, Service d'endocrinologie, diabétologie et maladies métaboliques, Clermont-Ferrand, France. ${ }^{3}$ Laboratoire GReD, Université Clermont Auvergne, Faculté de Médecine, Clermont Ferrand, France. ${ }^{4}$ Department of Molecular Medicine and Surgery, Karolinska Institutet, Stockholm, Sweden. ${ }^{5}$ Swedish Metabolomics Centre, Department of Forest Genetics and Plant Physiology, Swedish University of Agricultural Sciences, Umeå, Sweden. ${ }^{6}$ The NovoNordisk Foundation Centre for Basic Metabolic Research, Faculty of Health and Medical Sciences, University of Copenhagen, Copenhagen, Denmark. ${ }^{7}$ Department of Biosciences and Nutrition, Karolinska Institutet, Huddinge, Sweden. ${ }^{8}$ Metabolomics and proteomics core (MPC), Helmholtz Zentrum München, Neuherberg, Germany. ${ }^{9}$ Research Unit Analytical BioGeoChemistry, Helmholtz Zentrum München, Neuherberg, Germany. ${ }^{10} \mathrm{Chair}$ of Analytical Food Chemistry, TUM School of Life Sciences, Freising, Germany. "Department of Biosciences and Nutrition, Karolinska Comprehensive Cancer Center, Karolinska Institutet, Huddinge, Sweden. ${ }^{12}$ Radcliffe Department of Medicine, University of Oxford, Oxford, UK. ${ }^{13}$ Department of Physiology and Pharmacology, Karolinska Institutet, Stockholm, Sweden. ${ }^{14}$ These authors contributed equally: Salwan Maqdasy, Simon Lecoutre. ${ }^{15}$ These authors jointly supervised this work: Niklas Mejhert, Mikael Rydén.凶e-mail: niklas.mejhert@ki.se; mikael.ryden@ki.se 


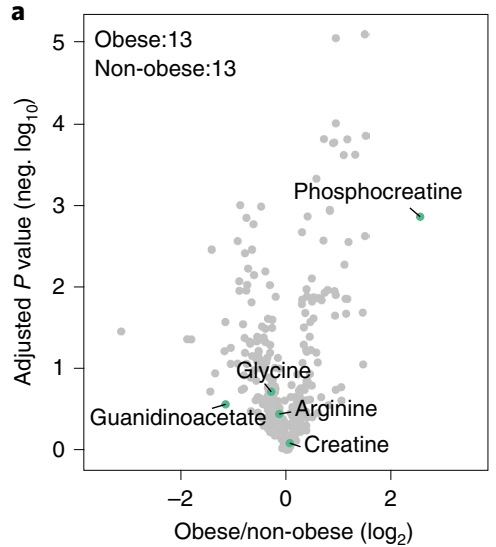

d

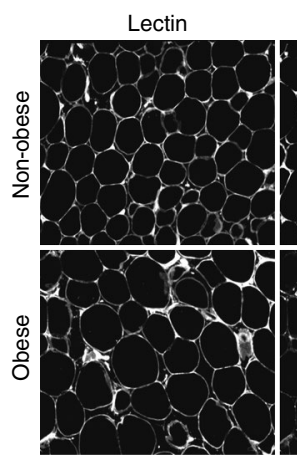

b

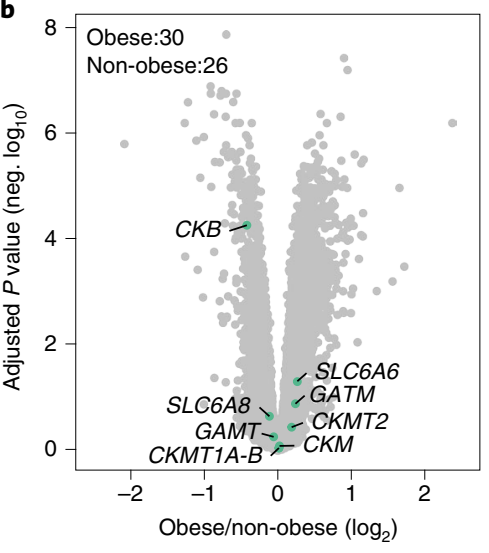

c

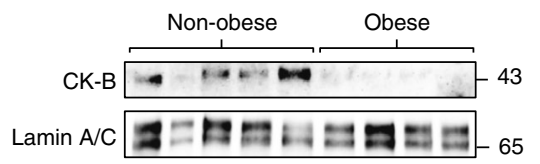

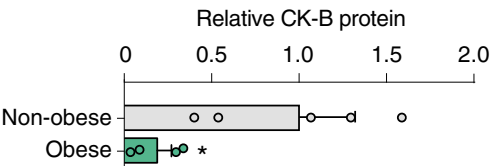
2.0 Obese $-\infty+-\infty$ *

Fig. 1 | Obesity is associated with altered phosphocreatine/creatine metabolism in human WAT. a, Polar metabolites in subcutaneous WAT of obese $(n=13)$ and non-obese (NO, $n=13)$ subjects (cohort 1) highlighting metabolites in the phosphocreatine/creatine pathway (green dots). Data are represented in a volcano plot with fold changes $\left(\log _{2}\right)$ and adjusted $P$ values (negative (neg.) $\log _{10}$ ). Statistics were calculated by Welch's two-sample $t$-test followed by false discovery rate (FDR) correction for multiple comparisons ( $q$ value), according to standard procedures from Metabolon. $\mathbf{b}$, Expression of genes encoding proteins in the phosphocreatine/creatine pathway in subcutaneous WAT of obese $(n=30)$ and non-obese $(n=26)$ women (cohort 2). Data are represented in a volcano plot with fold changes $\left(\log _{2}\right)$ and adjusted $P$ values (neg. $\log _{10}$ ) calculated using Limma (linear models for microarray and RNA-seq analysis). c, Western blot analysis of CK-B in subcutaneous WAT of obese $(n=4)$ and non-obese $(n=5)$ subjects. Lamin A/C was used as a loading control. ${ }^{\star} P=0.036$ by Student's two-sided $t$-test. $\mathbf{d}$, Representative immunofluorescence microphotographs of subcutaneous WAT from obese $(n=3)$ and non-obese $(n=3)$ subjects. Sections were stained with Lens culiniaris agglutinin (Lectin) and antibodies targeting CK-B. Scale bar, $50 \mu \mathrm{m}$. e, Creatine kinase activity was measured in total subcutaneous WAT ( $n=4$ from obese and $n=4$ non-obese subjects) as well as isolated mature adipocytes ( $n=5$ from obese and $n=7$ non-obese subjects). As illustrated in the upper panel, the creatine kinase activity measured in this assay represents the reverse reaction after addition of ADP where ATP is generated from phosphocreatine. Lower CK-B activity is expected to result in attenuated ATP levels. ${ }^{\star} P=0.05$; ${ }^{\star \star} P=0.003$ by Student's two-sided $t$-test. $\mathbf{f}$, Overview of the phosphocreatine/creatine pathway highlighting the alterations in human subcutaneous WAT linked to obesity. Data are represented as fold changes obese versus non-obese $\left(\log _{2}\right)$. ${ }^{*}$ Significant. In $\mathbf{c}$ and $\mathbf{e}$, data are shown as mean \pm s.e.m. CK-B, cytokine B; MA, mature adipocytes.

from glycine and arginine via the mitochondrial glycine amidinotransferase $(\mathrm{GATM})^{15,20}$. Guanidinoacetate is converted to creatine through guanidinoacetate $N$-methyltransferase (GAMT).

To determine if the alterations in phosphocreatine metabolism were reflected at the gene expression level, we analysed transcriptomic data of subcutaneous abdominal WAT from women with $(n=30)$ or without $(n=26)$ obesity (cohort 2, described in ref. ${ }^{21}$ and Supplementary Table 1 ). We found that $C K B$ displayed markedly lower expression in the obese state (Fig. 1b). As displayed in Extended Data Fig. 1a-b, this was confirmed in two independent datasets, where $C K B$ levels were lower in 15 obese versus 15 never-obese women (cohort 3 , described in ref. ${ }^{22}$ and Supplementary Table 1, Trial registration no. NCT01785134) and 18 obese versus 17 non-obese men (cohort 4, Supplementary Table 1, Trial registration no. NCT01727245). In cohort 3, CKB expression levels were completely normalized in the obese subjects following weight loss induced by bariatric surgery (Extended Data Fig. 1a). Western blot and immunofluorescence analyses confirmed that CK-B levels were lower in WAT of obese versus non-obese subjects (Fig. 1c,d), which was mirrored by attenuated CK activity in lysates obtained from both intact WAT and isolated mature fat cells of non-obese and obese women (Fig. 1e). Altogether, our systematic analyses of metabolomic and transcriptomic data, as well as protein levels and creatine kinase activity measures in human WAT, demonstrate that phosphocreatine abundance is increased and that CK-B expression/activity is decreased in obesity (Fig. 1f).

Adipocyte $C K B$ depletion induces a pro-inflammatory response. To dissect which adipose resident cells contribute to the generation of phosphocreatine, we analysed transcriptomic data from fractionated human $\mathrm{WAT}^{23}$. This revealed that mature adipocytes predominatly express CKB, CKMT2, GAMT and SLC6A8, while GATM and 
SLC6A6 are primarily expressed in adipose tissue macrophages (Fig. 2a). As a result, human white adipocytes express most of the genes involved in phosphocreatine/creatine metabolism, an observation that is supported by recent data showing that murine white adipocytes supply breast cancer cells with creatine metabolites ${ }^{20}$. We then determined whether human adipose-derived multipotent mesenchymal DPP4 ${ }^{+}$progenitors (Extended Data Fig. 2a) differentiated into adipocytes are a suitable model system to study this pathway in vitro. Quantitative analyses of expression levels during differentiation showed that the adipocyte-enriched phosphocreatine/creatine metabolism genes, in particular CKB and CKMT2, were highly expressed in the differentiated state (Fig. 2b) allowing us to study the effects of phosphocreatine/creatine perturbations on white adipocyte phenotype in vitro.

As $C K B$ expression was decreased in the obese state, we performed $C K B$ knockdown experiments in human adipocytes. Targeting $C K B$ by RNA interference (RNAi) resulted in a marked reduction in $C K B$ messenger RNA (mRNA) abundance (Fig. 2c), CK-B protein levels (Fig. 2d) and attenuated CK activity (Fig. 2e). In addition, $C K B$ silencing increased phosphocreatine abundance and the phosphocreatine/creatine ratio (Fig. 2f) without affecting creatine levels (Fig. 2f). CKB knockdown did not alter adipogenesis (Extended Data Fig. 2b-e) or thermogenic gene expression (Extended Data Fig. 2b). As CK-B localizes to both cytosol and mitochondria in brown adipocytes ${ }^{17}$, we next determined the subcellular distribution of CK-B in human white adipocytes. Fractionation experiments revealed that $\mathrm{CK}-\mathrm{B}$ is cytosolic, and that $C K B$ depletion resulted in decreased CK-B protein and CK activity specifically in this fraction (Fig. 2g). In contrast, CKMT2 silencing resulted in reduced protein abundance and CK activity in mitochondria but not in the cytosol (Fig. 2g). In addition, CKMT2 depletion resulted in decreased phosphocreatine levels and phospocreatine/creatine ratio without altering creatine abundance (Extended Data Fig. 2f). From these results in human white adipocytes, we conclude that (1) $C K B$ is the most highly expressed CK, (2) CK-B resides in the cytosol and (3) CK-B downregulation results in increased cellular phosphocreatine/creatine ratio.

Given that alterations in bioenergetic states are associated with marked transcriptional changes ${ }^{24}$, we next determined the effects of $C K B$ depletion on global gene transcription in human white adipocytes. A principal component analysis showed that $C K B$ knockdown cells were clearly separated from control-transfected adipocytes (Fig. 2h). To identify obesity-perturbed pathways affected by reduced $C K B$ levels, we integrated these in vitro transcriptomic data with genes co-expressed with $C K B$ in WAT of the
56 non-obese and obese women in cohort 2. This approach revealed that pro-inflammatory pathways were upregulated by $\operatorname{siCKB}$ in vitro and linked to low $C K B$ expression in vivo (Extended Data Fig. 2g). Analyses of individual genes within the pro-inflammatory pathways showed that CCL2 (encoding the chemokine Monocyte Chemoattractant Protein-1) displayed the largest fold-change upon siCKB (Fig. 2i). As adipocyte CCL2 secretion is increased in obesity and promotes WAT inflammation in both humans and animal model ${ }^{25-28}$, we focused our subsequent analyses on this chemokine. We validated the microarray findings by quantitative PCR (qPCR; Fig. 2j) and ELISA (Fig. 2k) and thus confirmed that CKB depletion upregulated both CCL2 gene expression and CCL2 protein secretion. The data above were obtained in cells from a male donor. However, similar effects were observed in differentitated adipocytes derived from a female donor (Extended Data Fig. 2h,i). The clinical relevance of these findings was further supported by multiple regression analyses in cohort 2 where CCL 2 mRNA levels (Fig. 2l), CCL2 secretion (Fig. $2 \mathrm{~m}$ ) and high sensitivity CRP levels (a measure of inflammation at the whole-body level, standardized $\beta=-0.40$, $P=0.0088$, graph not shown) were negatively associated with $C K B$ expression after correction for body mass index (BMI).

$C K B$ depletion regulates CCL2 through mitochondrial activity. Creatine metabolism regulates the bioenergetic state of cells ${ }^{29}$ and the latter is linked to inflammation and immune cell activation $^{30-33}$. As a result, we hypothesized that the association between $C K B$ depletion and CCL2 production is mediated by altered energy balance. We tested this by performing functional analyses of mitochondrial activity. Following carbonyl cyanide- $p$-trifluoromethoxyphenylhydrazone (FCCP)-titration experiments, we found that the oxygen consumption rate (OCR) was increased following $C K B$ knockdown (Fig. 3a and Extended Data Fig. 3a); this effect was accompanied by increased mitochondrial ATP production and cellular ATP/ADP ratio (Fig. 3b,c and Extended Data Fig. 3b), while mitochondrial abundance and morphology remained unchanged (Extended Data Fig. 3c-f). To determine if the perturbations in mitochondrial ATP production observed on $C K B$ depletion are required to upregulate CCL2 expression, we incubated siC-and siCKB-transfected adipocytes with or without oligomycin (an ATP synthase inhibitor) or bongkrekic acid (an adenine nucleotide translocase inhibitor) (Fig. 3d). Both inhibitors prevented the increase in ATP/ADP ratio (Fig. 3e) and CCL2 expression/secretion induced by $C K B$ RNAi (Fig. 3f,g), suggesting that changes in ATP/ADP ratio are required for mediating the effects of $C K B$ depletion on $C C L 2$ expression.

Fig. 2 | CKB silencing induces a pro-inflammatory profile in human white adipocytes. a, Expression of indicated genes in cells of human subcutaneous WAT. Results are displayed as z-scores. b, Expression of indicated genes during adipogenesis. c, CKB mRNA levels in human adipocytes (six replicates per condition, repeated three times) transfected with non-silencing ( $\mathrm{siC}$ ) or $C K B$-targeting (siCKB) oligonucleotides. ${ }^{\star \star \star \star} P<0.0001$. d, Western blot displaying CK-B and glyceraldehyde 3-phosphate dehydrogenase (GAPDH) in human adipocytes (three replicates per condition, repeated three times) transfected with siC or siCKB. ${ }^{\star \star \star} P=0.0002$. e, Creatine kinase activity measured in lysates of human adipocytes (three to four replicates per condition, repeated three times) transfected with siC or siCKB. ${ }^{* \star} P=0.0003$. f, Metabolite levels in human adipocytes (ten replicates per condition, repeated twice) transfected with siC or siCKB. ${ }^{\star \star} P=0.0093$ for phosphocreatine and 0.002 for phosphocreatine/creatine ratio. $\mathbf{g}$, Western blot showing the protein levels in mitochondrial and cytoplasmic fractions of human adipocytes transfected with siC, siCKB or siCKMT2 (repeated three times). Creatine kinase activity was determined in paired lysates as indicated in the right panel from one experiment. $\mathbf{h}$, Principal component analysis based on microarray data from human adipocytes (three replicates per condition) transfected with $\mathrm{siC}$ or siCKB. Ellipses indicate $95 \%$ confidence intervals. $\mathbf{i}$, log ${ }_{2}$ (fold-change) of genes regulated by siCKB in human adipocytes (upper half, from microarray data presented in $\mathbf{h}$ ). For each gene, the association (Spearman's rank correlation coefficient $\rho$ value) with CKB expression in WAT transcriptomic data from cohort 2 is shown (lower half). Leading edge genes in the GSEA 'HALLMARK_INFLAMMATORY_RESPONSE' pathway identified in Extended Data Fig. $2 \mathrm{~g}$ are shown. $\mathbf{j}$, CCL2 mRNA expression in human adipocytes (six replicates per condition, repeated three times) transfected with $\mathrm{siC}$ or siCKB. ${ }^{\star \star \star} P=0.0002$. $\mathbf{k}$, CCL2 secretion measured by ELISA from human adipocytes (three replicates per condition, repeated three times) transfected with siC or siCKB. ${ }^{\star \star} P=0.0059$. I, Correlation between CCL2 and CKB mRNA levels in human WAT from cohort 2. $\mathbf{m}$, Correlation between CCL2 WAT secretion and CKB mRNA levels in human WAT from cohort 2. In $\mathbf{c}-\mathbf{f}$, $\mathbf{j}$ and $\mathbf{k}$, Student's two-sided $t$-test was used. In $\mathbf{I}$ and $\mathbf{m}$, standardized $\beta$ and $P$ values are shown for multiple regression analysis after BMI correction. Data in $\mathbf{c}-\mathbf{f}, \mathbf{j}$ and $\mathbf{k}$ are shown as mean \pm s.e.m. APC, adipocyte progenitor cells; ATM, adipose tissue macrophages; cyto, cytoplasm; Cr, creatine; M1, M1-macrophages; M2, $\mathrm{M} 2$-macrophages; mito, mitochondria, $\mathrm{PC}$, principal component; $\mathrm{PCr}$, phosphocreatine. 
CCL2 induction upon $C K B$ depletion requires glucose usage. We next determined whether the link between the $\mathrm{ATP} / \mathrm{ADP}$ ratio and CCL2 expression upon CKB knockdown was dependent on a specific macronutrient. We studied acute changes in OCR of control or $C K B$-silenced white adipocytes treated with inhibitors of mitochondrial pyruvate transport
(2-cyano-3-(1-phenyl-1H-indol-3-yl)-2-propenoic acid (UK5099)), glutaminase (bis-2-(5-phenylacetamido-1,3,4-thiadiazol-2-yl)ethyl sulfide (BPTES)) or mitochondrial fatty acid translocation (Etomoxir (ETO)) (Fig. 3d). UK5099, not BPTES or ETO, impaired the rise in OCR of $C K B$-depleted white adipocytes (Fig. 4a). This suggests that $C K B$ knockdown increases glucose-derived a

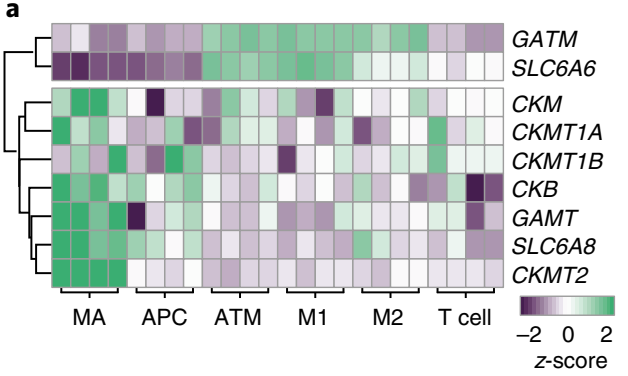

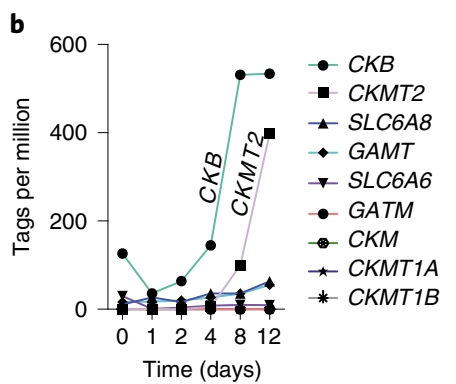

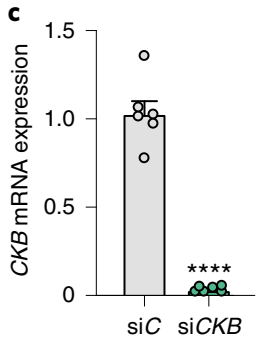

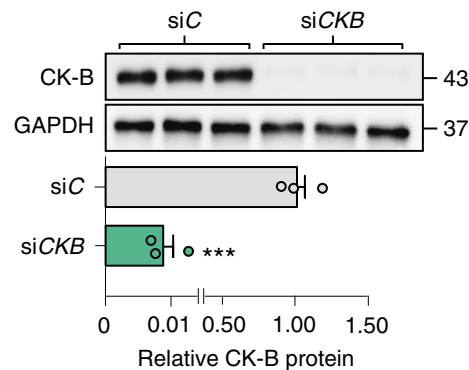

$\mathbf{g}$

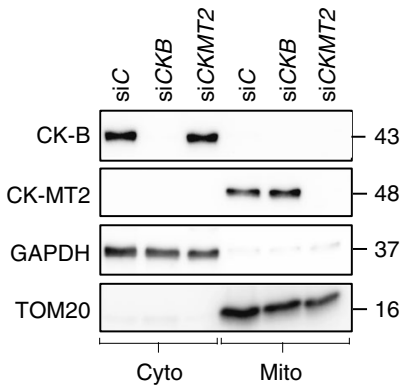

e

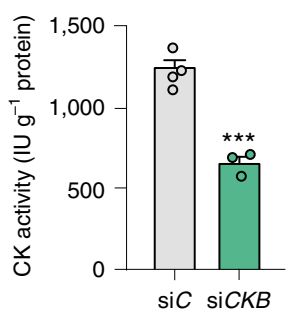

f

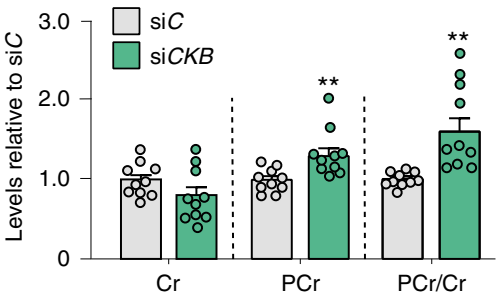

h
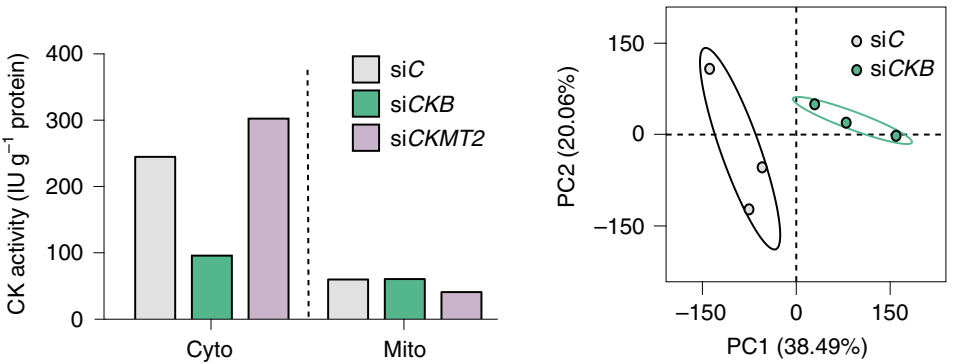

i
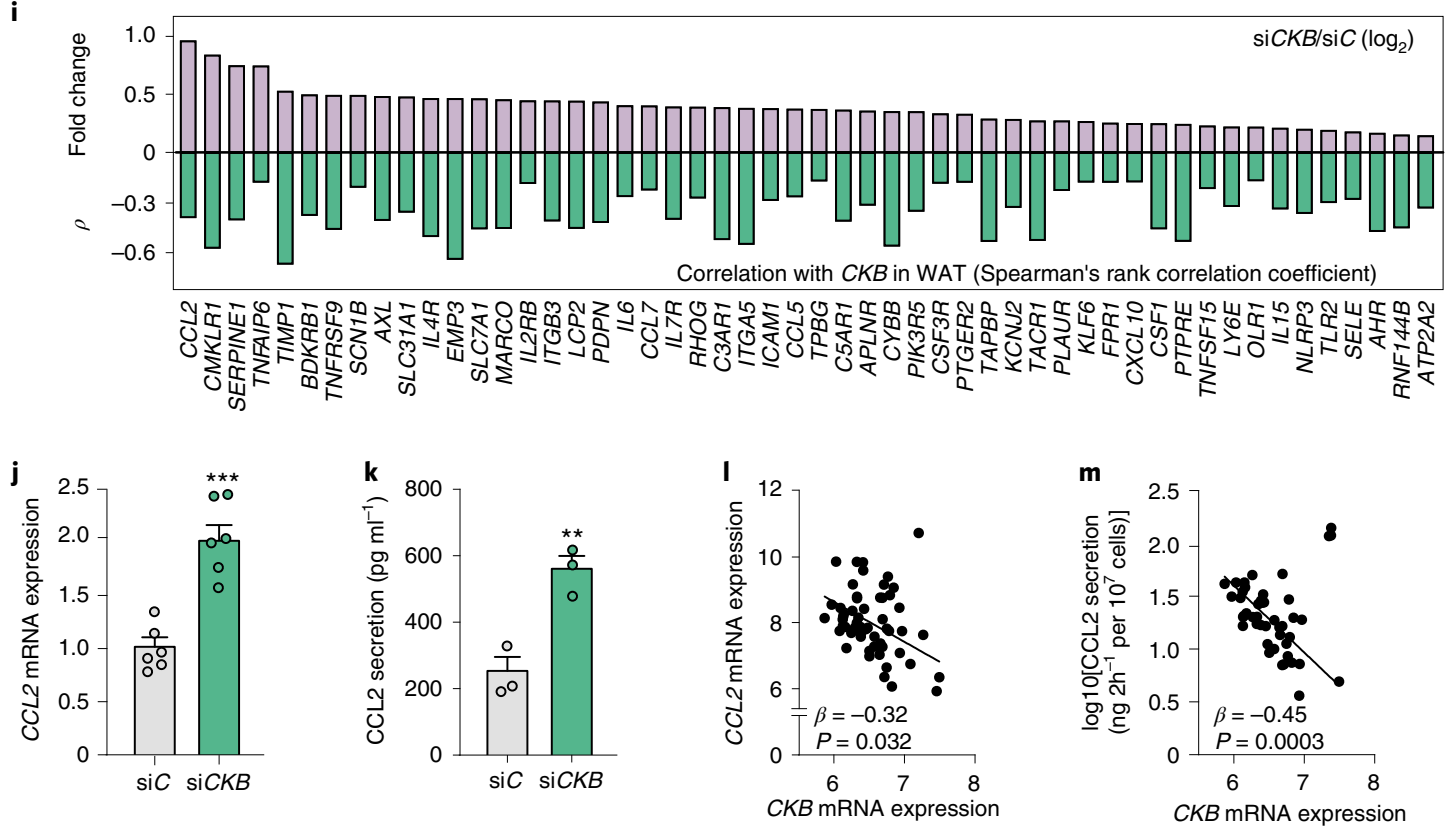


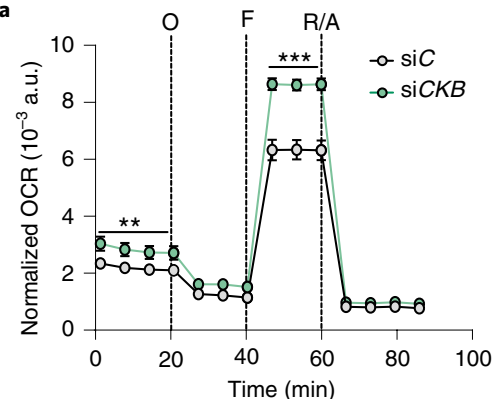

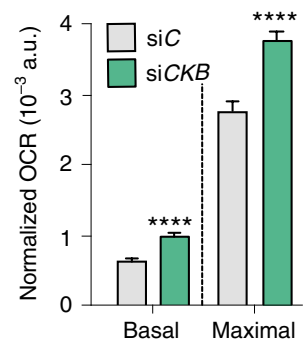

b

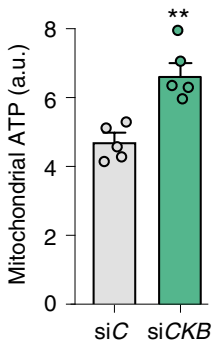

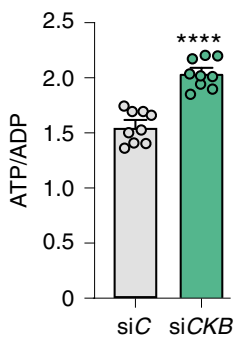

d

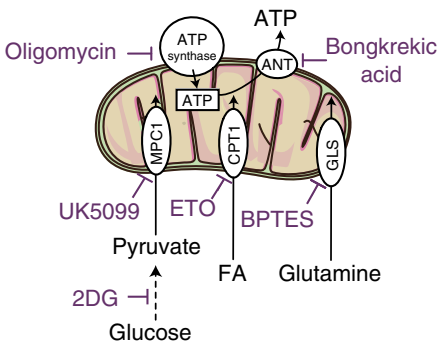

e

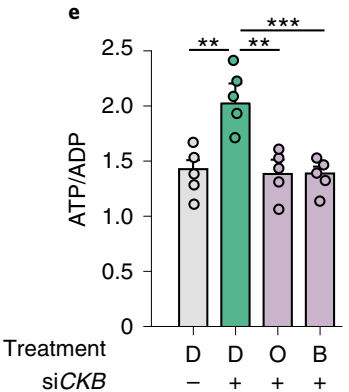

$\mathbf{f}$

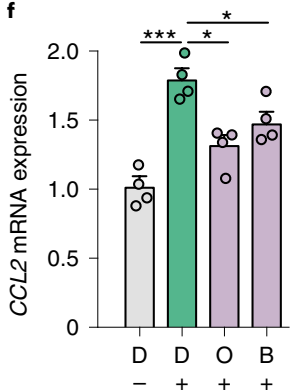

g

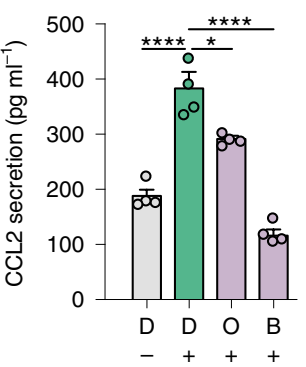

Fig. 3 | Adipocyte CK-B regulates CCL2 expression by modulating mitochondrial energy production. a, Normalized OCR determined in human adipocytes transfected with scrambled non-silencing ( $\mathrm{siC}$ ) or CKB-targeting ( $\mathrm{siCKB}$ ) oligonucleotides (12 replicates per condition, repeated three times). Non-mitochondrial respiration was substracted from the basal and maximal respiration is represented in the bar $c h a r t$. ${ }^{\star \star} P=0.0059,{ }^{\star \star \star} P=0.0008$, ${ }^{\star \star \star \star} P<0.0001$ (both basal and maximal respiration). O, Oligomycin; F, FCCP; R/A, rotenone/antimycin. b, Mitochondrial ATP production based on Seahorse data in human adipocytes transfected with $\mathrm{siC}$ or $\mathrm{siCKB}$ ( $n=$ five replicates per condition, repeated twice). ${ }^{\star \star} P=0.0078$. $\mathbf{c}$, ATP/ADP ratio measured by bioluminescence in human adipocytes transfected with siC or siCKB (nine replicates per condition, repeated twice). $\star_{\star} \times \star P<0.0001$. d, Schematic representation of drugs targeting mitochondrial ATP production and/or substrate usage. e, Effect of the mitochondrial inhibitors $1 \mu M$ oligomycin (O) or $1 \mu \mathrm{M}$ bongkrekic acid (B) for $24 \mathrm{~h}$ on ATP/ADP ratio in human adipocytes transfected with siC or siCKB. Control wells were treated with dimethyl sulfoxide (DMSO) (D) (five replicates per condition, repeated twice). Overall $P=0.043$. f, Same experimental setup as e but displaying CCL2 mRNA expression (four replicates per condition, repeated twice). Overall $P=0.0003$. $\mathbf{g}$, Same experimental setup as in e but displaying CCL2 secretion $\left(\mathrm{pg} \mathrm{m} \mathrm{m}^{-1}\right.$ ) detected by ELISA (four replicates per condition, repeated twice). Overall $P<0.0001$. Data were analysed by Student's two-sided $t$-test in a-c and by one-way ANOVA in e-g (Tukey's post-hoc tests indicated by ${ }^{\star} P<0.05,{ }^{\star \star} P<0.01,{ }^{\star \star \star} P<0.001,{ }^{\star \star \star \star} P<0.0001$ ). Data are shown as mean \pm s.e.m. 2DG, 2-deoxy-D-glucose; ANT, adenine nucleotide translocase; a.u., arbitray unit; CPT1, carnitine palmitoyl transferase 1; FA, fatty acid; GLS, glutaminase; MPC1, mitochondrial pyruvate carrier 1.

carbon utilization, a notion supported by the observation that glucose uptake and intermediates in the glycolytic pathway (glucose, glucose-6-phosphate and lactate) were increased in CKB-depleted cells (Fig. 4b,c). We further tested the link between increased glucose utilization and CCL2 by comparing the effects of UK5099 and the hexokinase inhibitor 2-deoxy-D-glucose (2DG). Both inhibitors prevented the induction of OCR (Fig. 4d), the rise in the ATP/ADP ratio (Fig. 4e), as well as elevation of CCL2 mRNA expression and secretion (Fig. 4f,g) upon $C K B$ silencing. In contrast, the elevation of CCL2 expression upon CKB silencing was unaltered by ETO and BPTES treatment (Extended Data Fig. 4a,b). Together, these data suggest that the the import of glucose-derived carbons into mitochondria is required for activating inflammatory gene expression upon $C K B$ silencing in white adipocytes.

Reduction in AMPK activity induces CCL2 expression. To test whether the effect of $C K B$ depletion on mitochondrial activity was triggered by a reduced ability to generate ATP from phosphocreatine and/or an increase in phosphocreatine abundance, we incubated human adipocytes with phosphocreatine or creatine. While incubations with creatine had no effects on OCR, ATP/ADP ratio or CCL2 expression (Extended Data Fig. 5a-c), the addition of phosphocreatine increased CCL2 expression (Fig. 5a), intracellular phosphocreatine levels (Fig. 5b) and the ratio of ATP/ADP (Fig. 5c), However, in contrast to $C K B$ depletion, phosphocreatine incubation did not affect OCR (Fig. 5d). To determine whether the observed effects were due to phosphocreatine uptake, we silenced SLC6A8. This prevented the increase in intracellular phosphocreatine levels as well as CCL2 expression and secretion following addition of phosphocreatine (Fig. 5e-h). Furthermore, phosphocreatine had no additive effects on OCR, ATP/ADP ratio and CCL2 expression in siCKB-transfected cells (Extended Data Fig. 5d-f). Taken together, our results indicate that the effects of both $\mathrm{siCKB}$ and phosphocreatine incubations on CCL2 expression converge on increases in the ATP/ADP ratio, while alterations in mitochondrial activity are only linked to $C K B$ depletion.

As AMPK senses the ATP/AMP ratio and is a well-established regulator of inflammatory responses (as reviewed in ref. ${ }^{34,35}$ ), we hypothesized that decreased AMPK activity could mediate the effects on CCL2 observed in CKB-depleted and phosphocreatine-treated adipocytes. To determine if AMPK impacts inflammation in white adipocytes, we silenced AMPK subunits $\alpha 1$ (encoded by PRKAA1) and $\gamma 1$ (PRKAG1) by RNAi. This resulted in reduced levels of AMPK abundance/activity (based on phosphorylation of Threonine 172) and increased CCL2 mRNA expression/secretion (Fig. 6a-d). In contrast, the pan-AMPK activator PF-739 stimulated AMPK activity without affecting the levels of $\mathrm{CK}-\mathrm{B}$, and abrogated the induction of CCL2 transcription by tumour necrosis factor alpha (TNF- $\alpha$ ), a canonical positive regulator of inflammation (Extended Data Fig. $6 \mathrm{a}, \mathrm{b})^{36,37}$. After establishing a causal link between AMPK and CCL2 expression in human white adipocytes, we next measured AMPK phosphorylation in cells transfected with $\mathrm{siCKB}$ or incubated with 

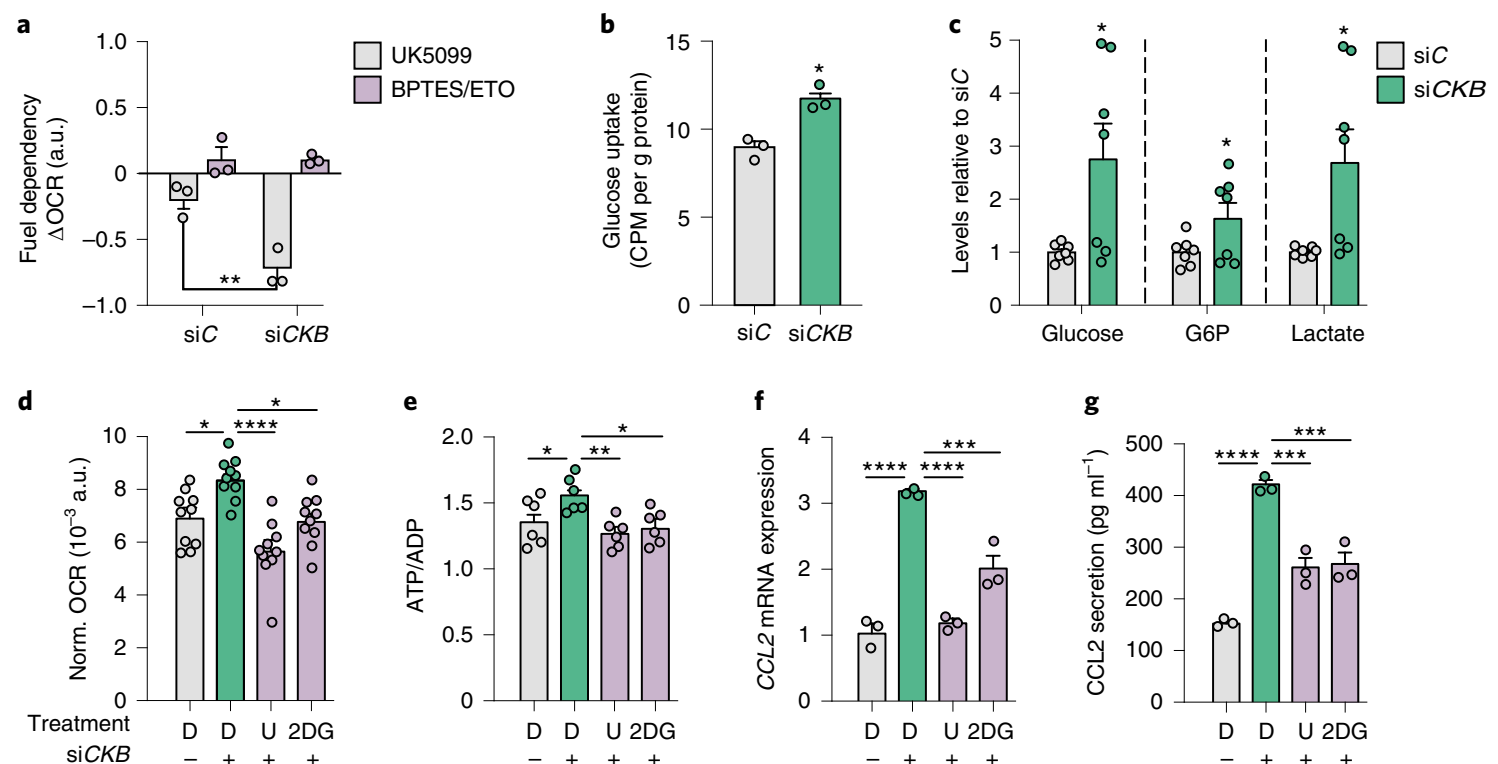

\begin{abstract}
Fig. 4 | Mitochondrial activation upon CKB downregulation is driven by increased glycolytic activity and glucose uptake. a, Delta OCR determined by mitochondrial fuel flex test using drugs targeting mitochondrial substrate usage (glycolysis, fatty acid oxidation and glutaminolysis) in human adipocytes transfected with siC or siCKB (three replicates per condition, repeated twice). Overall $P=0.0099$. b, Glucose uptake levels in human adipocytes transfected with siC or siCKB (three replicates per condition, repeated twice). ${ }^{\star} P=0.023$. c, Intracellular levels of glucose, glucose-6-phosphate and lactate in human adipocytes transfected with siC or siCKB (seven replicates per condition). ${ }^{\star} P=0.02,0.049$ and 0.02 , respectively (from left to right). d. Normalized OCR in human adipocytes transfected with siC or siCKB and incubated with $0.1 \mathrm{mmol} \mathrm{I}^{-1} 2 \mathrm{DG}$ or $10 \mu \mathrm{mol} \mathrm{I}^{-1}$ of UK5099 (U) or DMSO (D) for $24 \mathrm{~h}$ (ten replicates per condition). Overall $P<0.0001$. e, Same experimental setup as in $\mathbf{d}$ but displaying the effects on ATP/ADP ratio (six replicates per condition). Overall $P=0.0048$. f, Same experimental setup as in $\mathbf{d}$ but displaying the effects on CCL2 mRNA expression (three replicates per condition, repeated twice). Overall $P<0.0001$. $\mathbf{g}$, Same experimental setup as in $\mathbf{d}$ but displaying the effects on CCL2 secretion (pg $\left.\mathrm{ml}^{-1}\right)$ (three replicates per condition, repeated twice). Overall $P<0.0001$. Data were analysed by Student's two-sided $t$-test in $\mathbf{b}$ and $\mathbf{c}$, by one-way ANOVA in $\mathbf{d}$-g and by two-way ANOVA in a (Tukey's post-hoc tests indicated by $\left.{ }^{\star} P<0.05,{ }^{\star \star} P<0.01,{ }^{\star \star \star} P<0.001,{ }^{\star \star \star \star} P<0.0001\right)$. Data are shown as mean \pm s.e.m. CPM, counts per minute; G6P, glucose-6-phosphate; norm., normalized.
\end{abstract}

phosphocreatine. Compared to control cells, AMPK phosphorylation was reduced in both conditions (Fig. 6e,f). To test if the effects of $C K B$ depletion on CCL2 expression depend on reduced AMPK activity, we incubated siCKB- and siC-transfected cells with and without PF-739. Our data provide evidence that PF-739 abrogated the siCKB-induced increase in CCL2 mRNA expression (Fig. 6g), indicating that AMPK links reduced CK-B to increased CCL2 production. To exclude potential off-target effects, we also tested additional AMPK agonists, including 5-aminoimidazole-4-carboxamide riboside and metformin (Extended Data Fig. 6c,d), as well as another short interfering RNA (siRNA) targeting $C K B$ (Extended Data Fig. 6e-h). These experiments reproduced the effects of $C K B$ depletion and AMPK activation, further supporting the idea that perturbations in phosphocreatine/creatine metabolism affect CCL2 expression through reduced AMPK activity in white adipocytes.

Murine in vivo models corroborate human findings. To assess the relevance of our findings in vivo, we analysed published datasets and investigated different mouse models. First, we mined single-nucleus transcriptomics of epididymal WAT ${ }^{38}$ and observed that, similar to humans, $C k b$ is the most highly expressed creatine kinase-encoding gene in white adipocytes (Extended Data Fig. 7a). As obese humans display decreased $C K B$ expression and increased phosphocreatine levels, we next determined if a short (five weeks) high fat diet (HFD) regimen recapitulated this phenotype in mice. This intervention length was chosen to determine if alterations in phosphocreatine/creatine metabolism are early events in the development of obesity. As expected, HFD-feeding resulted in increased body weight and fat mass (Extended Data Fig. 7b,c), impaired glucose tolerance (Extended Data Fig. 7d) and WAT inflammation with increased gene expression of $\mathrm{Ccl} 2$, as well as the macrophage markers Cd68 and Adgre1 (F4/80), where the latter was confirmed by immunofluorescence analysis (Extended Data Fig. 7e,f). Following this general characterization, we measured CK-B mRNA/protein and phosphocreatine/creatine levels in WAT. We found that mice fed HFD exhibited lower levels of Ckb mRNA (Fig. 7a) and CK-B protein, where the latter was observed both in total tissue lysates and in intact tissue by immunofluorescence analysis (Fig. 7b,c). This was accompanied by higher phosphocreatine levels and phosphocreatine/creatine ratio compared to mice on chow diet (CD; Fig. $7 \mathrm{~d})$. In concordance with the findings in our clinical cohorts, these results confirm that the phosphocreatine/creatine pathway is perturbed by weight gain in mice. To determine if elevating phosphocreatine levels was sufficient to enhance WAT inflammation in vivo, we administered phosphocreatine $\left(3 \mathrm{mg} \mathrm{g}^{-1}\right)$ or phosphate buffered saline (PBS) intraperitoneally in chow-fed mice daily for seven days. This short-term treatment resulted in significant increases in WAT phosphocreatine levels (Fig. 7e) but did not influence body weight, WAT weight, fasting plasma glucose and insulin (Extended Data Fig. $7 \mathrm{~g}-\mathrm{j}$ ) or $C k b$ mRNA and protein levels (Fig. $7 \mathrm{f}, \mathrm{g}$ ). However, we observed an increase in the expression of $\mathrm{Ccl} 2$ and $\mathrm{Cd} 68$ (with a trend for an increase in Adgre1) (Fig. 7f) and increased immunofluorescence staining of $\mathrm{F} 4 / 80$ following phosphocreatine injections (Fig. 7h).

As intraperitoneal injections with high doses of phosphocreatine may affect multiple organs, we determined the effects of $C k b$ depletion on WAT inflammation in vivo by comparing adipocyte-specific $C k b$ deleted $\left(C k b^{\text {Adipoq-Cre }}\right)$ with control $\left(C k b^{f l / f l}\right)$ mice fed a HFD for 16 weeks. These samples were obtained from a published study where 

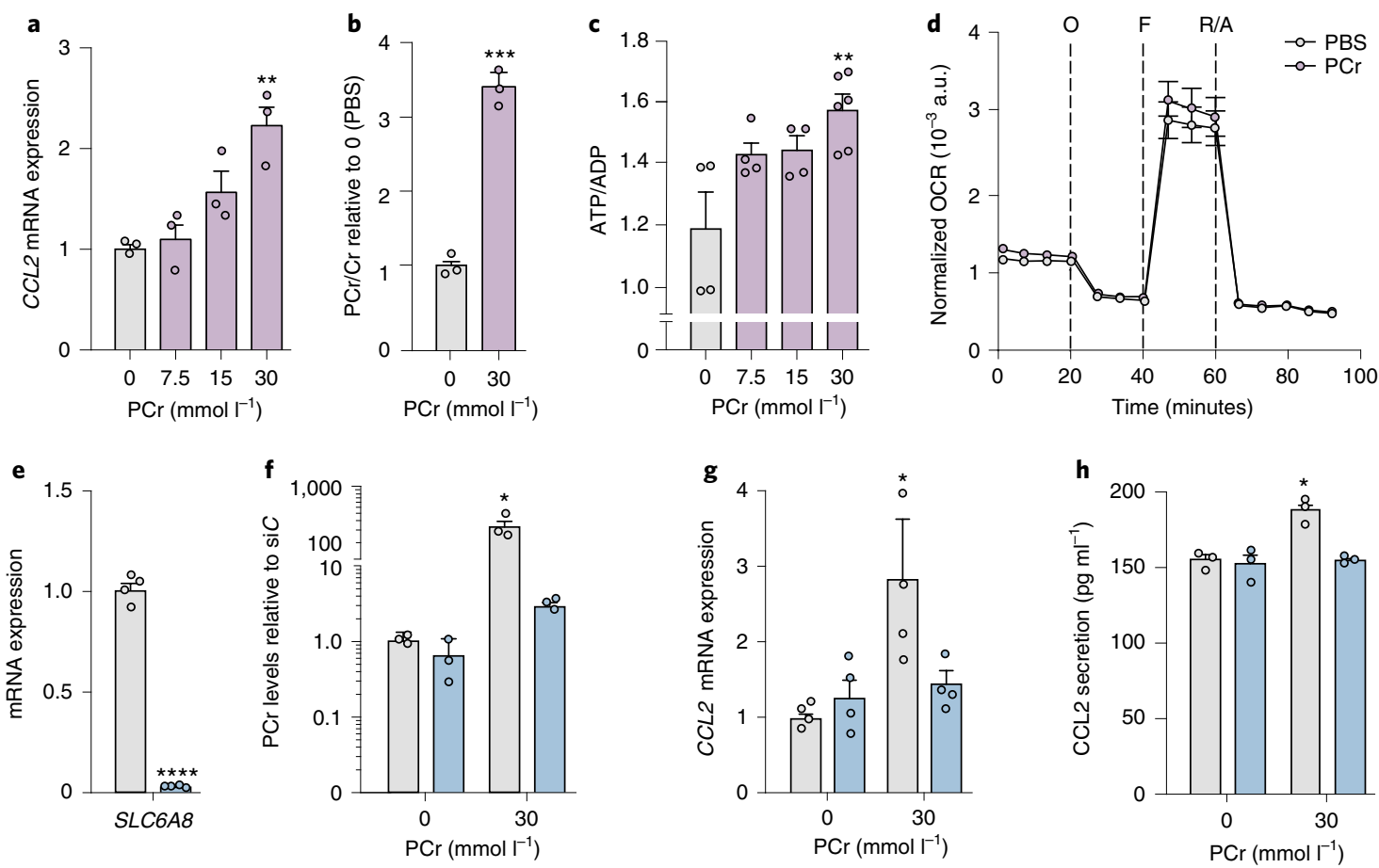

Fig. 5 | Phosphocreatine is taken up by SLC6A8 in white adipocytes and promotes CCL2 production. a, Human adipocytes were incubated with PBS or different concentrations of phosphocreatine for $24 \mathrm{~h}$ and effects on CCL2 mRNA expression were determined (three replicates per condition, repeated twice). Overall $P=0.0021$. b, The ratio of phosphocreatine/creatine in human in vitro differentiated adipocytes incubated with PBS or 30 mmol $\mathrm{I}^{-1}$ phosphocreatine for $24 \mathrm{~h}$ (three replicates per condition, repeated twice). ${ }^{\star \star \star} P=0.0002$. c , Same experiments as in a but displaying the ratio of ATP/ADP ratio (four replicates per condition for $0-15 \mathrm{mmol} \mathrm{I}^{-1}$ and six replicates per condition for $30 \mathrm{mmoll}^{-1}$, repeated twice). Overall $P=0.0068$. d, Normalized OCR in human adipocytes incubated with $30 \mathrm{mmol} \mathrm{I}^{-1}$ phosphocreatine for $24 \mathrm{~h}$ (six replicates per condition, repeated twice). e, Expression of SLC6A8 in human adipocytes transfected with scrambled non-silencing ( $\mathrm{siC}$ ) or SLC6A8-targeting (siSLC6A8) oligonucleotides (four replicates per condition, repeated three times). ${ }^{\star \star \star \star} P<0.0001$. f, Phosphocreatine levels in human adipocytes transfected with siC or siSLC6A8, and incubated in the presence or absence of $30 \mathrm{mmol} / \mathrm{L}$ phosphocreatine for $24 \mathrm{~h}$ (three replicates per condition, repeated twice). Overall $P=0.0028$. g, Same experimental setup as in $\mathbf{f}$ but displaying the effects on CCL2 mRNA levels (four replicates per condition, repeated twice). Overall $P=0.05$. $\mathbf{h}$, Same experimental setup as in $\mathbf{f}$ but displaying the effects on CCL2 secretion (three replicates per condition, repeated twice). Overall $P=0.05$. Data were analysed by Student's two-sided $t$-test in $\mathbf{b}$ and $\mathbf{e}$, by one-way ANOVA in $\mathbf{a}$ and $\mathbf{c}$ and by two-way ANOVA in $\mathbf{f}-\mathbf{h}$. Tukey's post-hoc tests indicated by ${ }^{\star} P<0.05$, ${ }^{\star \star} P<0.01$. Data are shown as mean \pm s.e.m.

adipocyte-specific $C k b$ depletion promoted obesity and glucose intolerance ${ }^{17}$. However, due to the higher food consumption of control mice at the end of the intervention, both groups of mice displayed similar body weight/composition although the $C k b^{\text {Adipoq-Cre }}$ mice were more glucose intolerant and insulin-resistant ${ }^{17}$. We confirmed that the $C k b^{\text {Adipoq-Cre }}$ animals expressed lower $C k b$ mRNA levels (Fig. 8a) and CK-B protein in WAT (Fig. 8b), which was accompanied by an increase in phosphocreatine levels and the phosphocreatine/creatine ratio without affecting creatine abundance (Fig. 8c). Transcriptional measurements showed that $\mathrm{Ccl} 2$, Adgre 1 and Cd68 mRNA levels were higher in WAT of $C k b^{\text {Adipoq-Cre }}$ mice compared to controls (Fig. 8d). We further validated the pro-inflammatory effect by performing immunofluorescence staining of F4/80. Our results show that macrophage infiltration was increased in the WAT of $C k b$-depleted animals versus control littermates (Fig. 8e). As the $C k b^{\text {Adipoq-Cre }}$ lack CK-B in both brown and WAT, and display a defect in brown adipose tissue thermogenesis $^{17}$, we next examined if WAT inflammation was secondary to impaired adipocyte thermogenesis. However, the expression of the thermogenic adipocyte-markers Ppargcla, Ucp1 and Dio2, was unaltered in the WAT of $C k b^{\text {Adipoq-Cre }}$ mice compared to control mice (Fig. 8f). Finally, we tested if $C k b$ depletion specifically in brown adipocytes results in WAT inflammation. Compared to $C k b^{f l f l}$ mice, $C k b^{\text {UcP1-CreERT2 }}$ HFD-fed mice did not show any alterations in $C k b, C c l 2$, Adgre 1 and $C d 68$ mRNA levels in WAT (Fig. 8g). Together, these data demonstrate that perturbed phosphocreatine/creatine metabolism in WAT is linked to inflammation in both mice and humans.

\section{Discussion}

The phosphocreatine/creatine energy shuttling system is highly conserved across species. Despite the fact that this pathway was discovered more than 180 years ago ${ }^{39}$, its physiological role in organs other than the brain and skeletal/cardiac muscle remains poorly understood. Creatine metabolism modulates multiple aspects of brown adipocyte and immune cell biology ${ }^{16}$. More specifically, creatine promotes thermogenesis through stimulation of mitochondrial ATP turnover in brown adipose tissue ${ }^{17,29}$ and has been linked to M2-polarization in macrophages ${ }^{18}$. While a recent study suggested that white adipocytes in breast tissue can supply neighbouring tumour cells with creatine ${ }^{20}$, the role of this pathway in WAT is unclear. Herein, we demonstrate that white adipocyte phosphocreatine/creatine metabolism is perturbed in the obese state of both humans and mice. This results in altered ATP/ADP levels, which in turn attenuate AMPK activity leading to increased transcription of multiple pro-inflammatory genes including the chemokine CCL2. Collectively, our data unravel a link between energy shuttling and inflammation in white adipocytes and suggest that this balance is perturbed in obesity. 
a
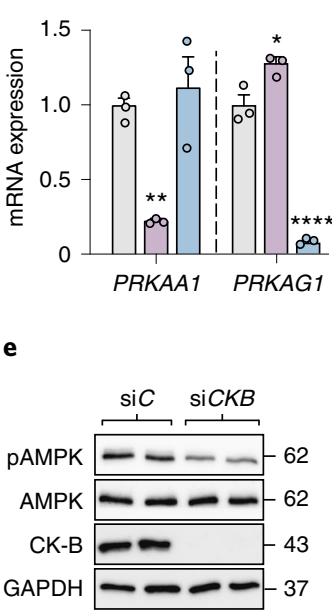

b

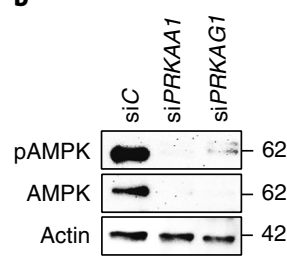

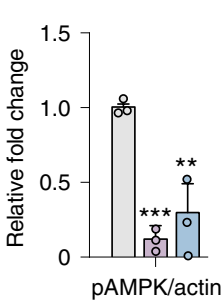

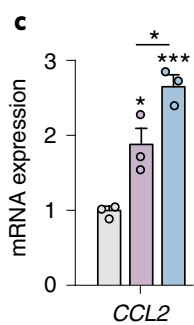

d

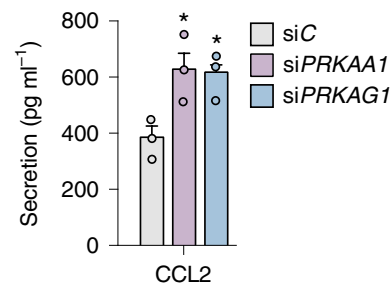

f

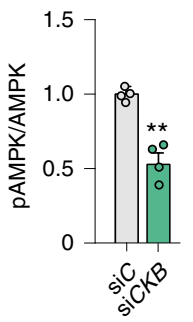

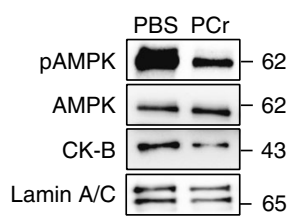

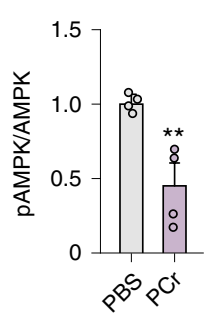

g

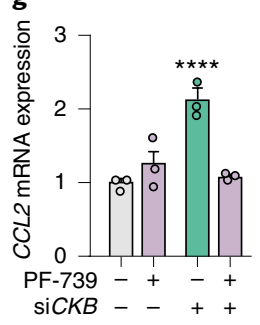

Fig. 6 | Perturbation in phosphocreatine metabolism is linked to CCL2 production via effects on AMPK activity. a, PRKAA1 and PRKAG1 mRNA expression in human adipocytes transfected with siPRKAA1, siPRKAG1 or siC (three replicates per condition, repeated twice). Overall $P=0.005$ for PRKAA1 and $P<0.0001$ for PRKAG1. b, AMPK, phosphorylated AMPK ( $\mathrm{PAMPK}$ ) and actin levels determined by western blot in human adipocytes transfected with siPRKAA1, siPRKAG1 or siC. The left panel shows representative blots and bar graphs to the right show quantifications of the indicated protein levels (three independent experiments). Overall $P=0.0013$. c, Same experimental setup as in a but displaying the effects on CCL2 mRNA levels (three replicates per condition, repeated twice). Overall $P=0.0011$. d, Same experimental setup as in a but displaying the effects on CCL2 secretion (three replicates per condition, repeated twice). Overall $P=0.0115$. e, AMPK, pAMPK, CK-B and GAPDH determined by western blot in human adipocytes transfected with $\mathrm{siC}$ or siCKB. The left panel shows representative blots and bar graphs to the right show quantifications of the indicated protein levels (two replicates per condition, repeated two times). ${ }^{\star \star} P=0.0018$. f, AMPK, pAMPK, CK-B and Lamin A/C determined by western blot in human adipocytes transfected with siC or siCKB, after incubation for $24 \mathrm{~h}$ with phosphocreatine $\left(30 \mathrm{mmol}^{-1}\right.$ ) or PBS. The left panel shows representative blots and bar graphs to the right show quantifications of the indicated protein levels (quantifications from four independent expirements). ${ }^{\star \star} P=0.0078$. $\mathbf{g}$, $C C L 2$ expression in human adipocytes transfected with $\mathrm{siC}$ or siCKB, after incubation with vehicle (DMSO) or PF-739 ( $5 \mu$ mol I-1) for $24 \mathrm{~h}$ (three replicates per condition, repeated twice). Overall $P<0.0001$. Data were analysed by Student's two-sided $t$-test in e and $\mathbf{f}$, by one-way ANOVA in panels a-d and two-way ANOVA in $\mathbf{g}$. Tukey's post-hoc tests indicated by ${ }^{\star} P<0.05,{ }^{\star \star} P<0.01,{ }^{\star \star \star} P<0.001,{ }^{\star \star \star \star} P<0.0001$. Data are shown as mean \pm s.e.m.

Creatine kinases constitute a family of enzymes interconverting phosphocreatine and creatine ${ }^{16}$. They are encoded by four distinct genes, whose products localize to mitochondria and/or cytosol and display different directionalities based on substrate availability, as well as cell type-specific expression patterns. Our systematic mapping of these enzymes shows that human white adipocytes predominatly express CK-B and CK-MT2, where the former localizes to the cytosol and the latter is present in mitochondria. As the reverse creatine kinase reaction is known to dominate in the cytosol, changes in CK-B activity alters the capacity to convert phosphocreatine to creatine. In human and murine obesity, the levels of CK-B in white adipocytes are selectively downregulated, which is linked to an increase in phosphocreatine levels and the phosphocreatine/creatine ratio. We further demonstrate that $C K B$ depletion in human white adipocytes mirrors this alteration and leads to an increase in glycolysis, mitochondrial activity and an accumulation of ATP. Our interpretation of these results is that perturbations in the creatine shuttle are sensed by the cells and result in compensatory changes in other bioenergetic pathways. The mechanisms linking these processes are currently unclear.

Evidence from studies of immune cells suggests that aerobic glycolysis and mitochondrial activity are closely related to inflammation and cytokine secretion ${ }^{30,40,41}$. As a result, we hypothesized that this constituted a link between $C K B$ depletion and the induction of a pro-inflammatory response. To test this model, we treated human white adipocytes and mice with phosphocreatine and observed a similar induction of several pro-inflammatory genes without changes in mitochondrial activity. Instead, the common denominator between $C K B$ depletion and phophocreatine incubation was an increase in the intracellular ATP/ADP ratio and an attenuation of AMPK activity. Treatments with multiple different AMPK activators abrogated the effects on CCL2 production induced by $C K B$ depletion/phosphocreatine incubation, suggesting that AMPK coordinates these pathways. In line with this, AMPK regulates inflammatory processes in adipose tissue $\mathrm{e}^{42,43}$ and we provide evidence that reduced AMPK activity (e.g., via knockdown of PRKAA1 and PRKAG1) increases CCL2 expression. In addition, AMPK activity is reduced in both obese rodents ${ }^{44}$ and humans ${ }^{45,46}$, and targeting this pathway has been proposed as a potential therapeutic avenue in metabolic disease ${ }^{47,48}$.

Previous studies have demonstrated cross-talks between phosphocreatine/creatine and AMPK in skeletal muscle ${ }^{49}$. More specifically, biochemical approaches revealed that an increased phosphocreatine/creatine ratio directly inhibits AMPK activity and that CK-M interacts physically with AMPK. Whether the latter pertains to CK-B is unclear, but our data revealed that AMPK activation abrogated the effects of $C K B$ depletion on CCL2 expression in white adipocytes. As a result, clinically, the beneficial effects of metformin in reversing insulin resistance may partly be mediated downstream of phosphocreatine accumulation in adipocytes. Metabolic comparisons of metformin and selective AMPK activation, as well as $C K B$ induction in experimental in vivo models of obesity may address this relationship. Unfortunately, at present, these studies are limited by the lack of specific $C K B$ regulators.

White adipocytes play a central role in regulating WAT inflammation by secreting a large number of pro-inflammatory cytokines and chemokines ${ }^{1}$. While a chronic release of these factors by adipocytes clearly results in immune cell infiltration and disturbed tissue 


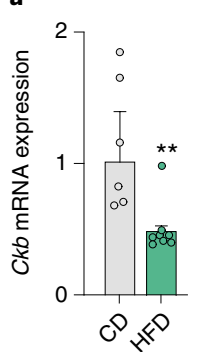

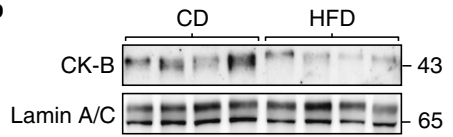

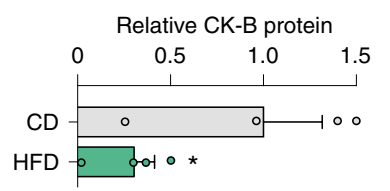

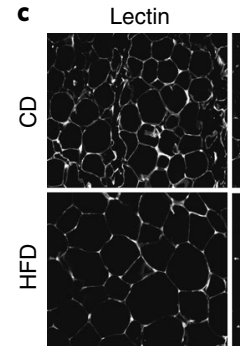

CK-B

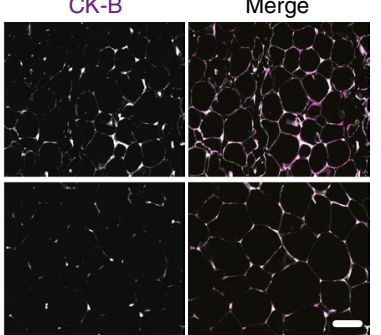

d

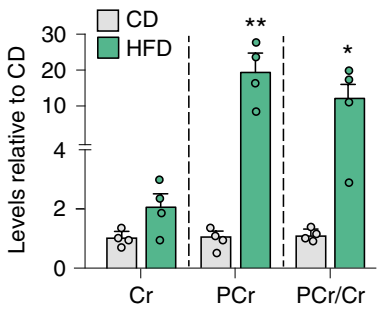

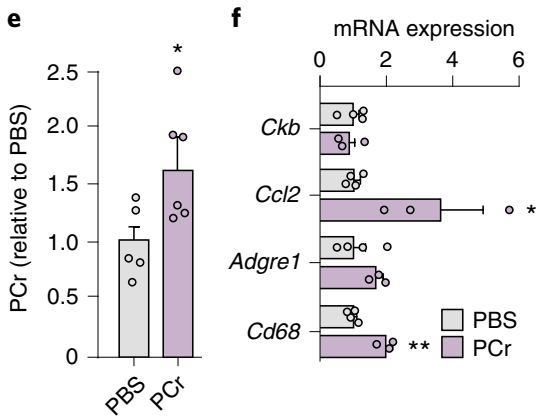

$\mathbf{g}$

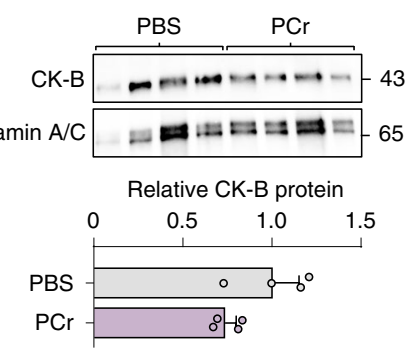

h

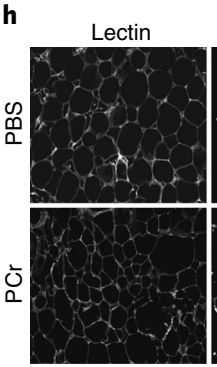

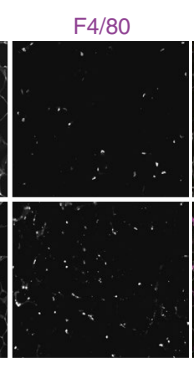

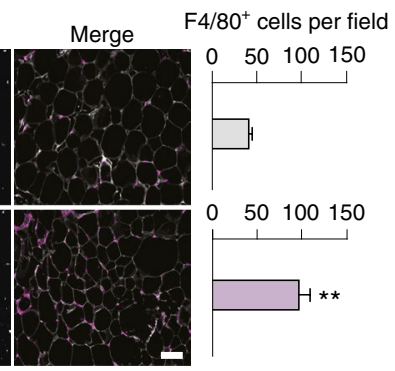

Fig. 7 | HFD and phosphocreatine injections promote WAT inflammation in vivo. a-d, Effect of HFD in male mice aged $10-11$ weeks on $C k b$ mRNA expression (six mice in CD and eight in HFD group). ${ }^{\star \star} P=0.0026$. (a) and CK-B protein abundance determined by western blot ( $n=4$ per group). ${ }^{\star} P=0.041$. (b) or immunofluorescence $(\mathbf{c})$ as well as phosphocreatine and creatine levels $\left(n=4\right.$ per group). ${ }^{\star} P=0.019$, ${ }^{\star \star} P=0.0063$. (d) in pgWAT. Scale bar, $50 \mu \mathrm{m}$. e, WAT phosphocreatine levels measured in 12-week-old male mice treated with either PBS or phosphocreatine injections ( 3 mg $g^{-1}$, intraperitoneally for 7 days, $n=5$ for PBS-injected and $n=6$ for phosphocreatine-injected mice). ${ }^{\star} P=0.049$. f, Transcriptional levels of $C k b$ and genes encoding pro-inflammatory markers/factors was determined in pgWAT ( $n=4$ for PBS-injected and $n=3$ for phosphocreatine-injected animals) of mice described in e. $P=0.029$ for $C c / 2$ and 0.0024 for $C d 68 . \mathbf{g}$, CK-B protein levels in pgWAT of mice injected with PBS or phosphocreatine ( $n=4$ per group). h, Immunofluorescence of F4/80 (magenta) and Lens culinaris agglutinin (Lectin, grey) in pgWAT of phosphocreatine- and PBS-injected mice. The number of $\mathrm{F} 4 / 80^{+}$cells was counted in three to four random fields of pgWAT per mouse in four mice. Scale bar, $50 \mu \mathrm{m}$. ${ }^{\star \star} P=0.0017$. Data were analysed by Student's two-sided $t$-test except for $\mathbf{b}$, which was analysed by Student's one-sided $t$-test. Data are shown as mean \pm s.e.m.

function, a transient adipocyte pro-inflammatory state is essential for tissue remodelling and healthy expansion ${ }^{50,51}$. However, despite decades of research, the signals triggering the pro-inflammatory response of white adipocytes remain largely unclear. Our present data suggest that the phosphocreatine/creatine system regulates adipocyte inflammation by intracellular energy shuttling. We speculate that $C K B$ downregulation in the obese state leads to phosphocreatine accumulation, perturbed intracellular energy metabolism, attenuated AMPK activity and the development of a chronic inflammatory response. The results presented herein and previously ${ }^{17}$ show that adipocyte-specific $C k b$ depletion results in obesity, WAT inflammation and insulin resistance. Importantly, the effects we report herein are not secondary to changes in brown adipose tissue thermogenesis or WAT beigeing as evidenced by expression analyses in CKB-depleted human adipocytes and WAT of $C k b^{\text {Adipoq-Cre }}$ and $C k b^{U c p 1-C r e E R T 2}$ mice. Altogether, our work highlights the importance of intracellular metabolites in coordinating metabolic and inflammatory processes in white adipocytes.

The factors controlling adipocyte $C K B$ transcription remain unknown. In other tissues, $C K B$ expression is regulated by adrenergic signalling and/or mechanical/oxidative stress ${ }^{17,52,53}$. Whether such regulatory events operate in white adipocytes remains unclear, but it is worth noting that all of these processes (for example, adipocyte death, hypoxia, ER stress and mechanical stress) are altered in obesity. Identification and perturbation of $C K B$ regulatory elements was beyond the scope of the present study. Instead, we used two different approaches, that is phosphocreatine treatment and genetic $C K B / C k b$ depletion models, to alter the balance of this system. Admittedly, the phosphocreatine concentrations used in cells/animals were higher than those observed in the circulation (reported to be in the $\mu \mathrm{M}$ range $\mathrm{r}^{54}$, which warrants caution in interpreting the data from these experiments. However, as a proof-of-concept these experiments indicate that the accumulation of phosphocreatine in WAT is sufficient to trigger the expression of downstream inflammatory genes. Finally, all studies in humans were performed in subcutaneous WAT and we cannot establish whether the same mechanisms are relevant in other WAT regions.

Based on a translational study design encompassing clinical cohorts and human as well as murine in vitro/in vivo models, we provide evidence that the phosphocreatine/creatine pathway is a determinant of pro-inflammatory status in white adipocytes. Our findings suggest that intracellular energy homeostasis is coordinated with WAT inflammation. Future studies may reveal whether nutritional/pharmacological interventions targeting phosphocreatine/creatine metabolism can improve the metabolic consequences linked to obesity.

\section{Methods}

Materials. Materials/reagents are listed with catalogue numbers and vendors in Supplementary Table 2.

Human subjects. Clinical data for all four cohorts are presented in Supplementary Table 1. All studies were approved by the regional ethics board and informed written consent was obtained from all participants.

Animal studies. For HFD and phosphocreatine injection studies, pathogen-free C57BL/6J male mice were purchased from Charles River (Germany). Mice were housed at the KM-B animal facility at the Karolinska Institutet in ventilated cages (four animals per cage) with a $12 \mathrm{~h}$ light/12 h dark cycle (lights on 06:00-18:00) in a temperature-controlled $\left(20-24^{\circ} \mathrm{C}, 50 \%\right.$ humidity) facility with ad libitum access to food and water. Animals were handled following the European Union laws and guidelines for animal care. Health inventories were performed on a 
a

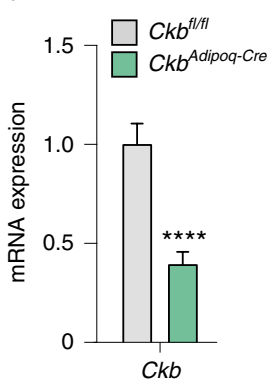

b

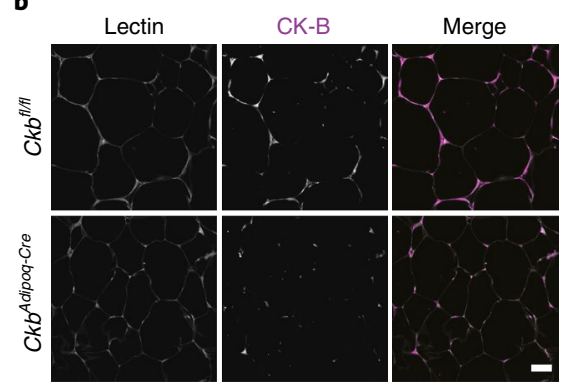

c

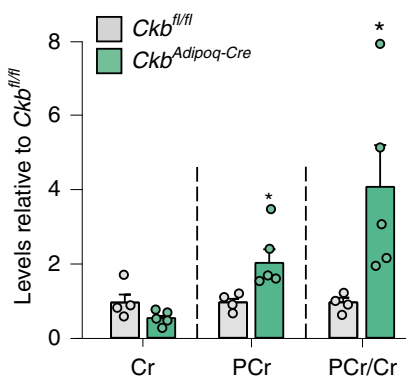

d

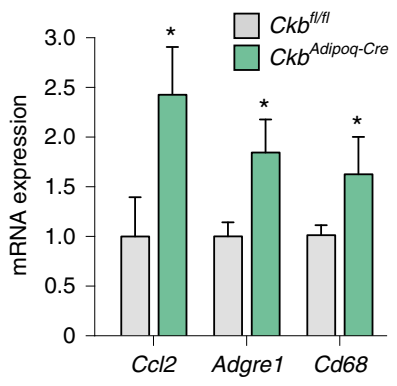

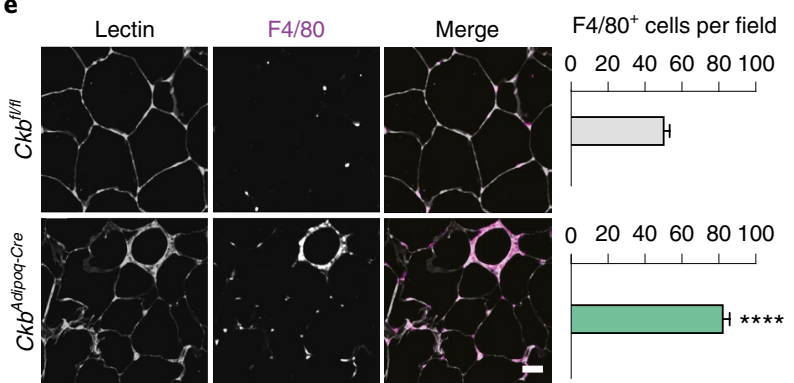

f

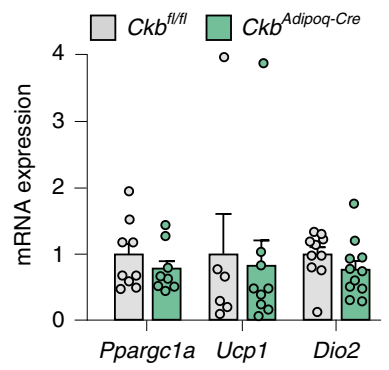

g

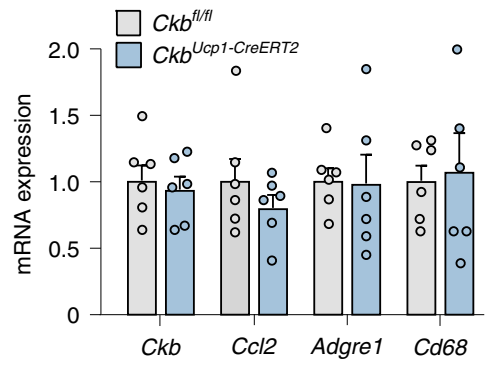

Fig. 8 | Adipocyte-specific $\mathbf{C} \boldsymbol{k} \boldsymbol{b}$ deletion induces WAT phosphocreatine accumulation and inflammation in vivo. a, Male mice with an adipocyte-specific deletion of $C k b\left(C k b^{\text {Adipoq-Cre }}\right)$ and control littermates $\left(C k b^{f / f l}\right)$ were fed a HFD for 16 weeks starting at four weeks of age. Effects on body weight and glucose tolerance of this cohort have been presented ${ }^{17}$. Ckb gene expression was determined in pgWAT by qPCR ( $n=11$ mice per group). ${ }^{\star \star \star \star} P<0.0001$. b, Representative immunofluorescence microphotographs of pgWAT from $C k b^{\text {Adipoq-Cre }}$ and $C k b^{f / f l}$ mice. Sections were stained with Lens culiniaris agglutinin (Lectin) and antibodies targeting CK-B. Scale bar, $50 \mu \mathrm{m}$. c, Phosphocreatine and creatine levels in pgWAT from $C k b^{\text {Adipoq-Cre }}(n=5)$ and $C k b^{f l / f l}(n=4)$ mice. ${ }^{\star} P=0.040$ for phosphocreatine and 0.045 for phosphocreatine/Cr, respectively. $\mathbf{d}$, mRNA levels for genes encoding inflammatory proteins in pgWAT from $C k b^{\text {Adipoq-Cre }}$ and $C k b^{f / f l}$ mice ( $n=11$ per group). $P=0.018$ for $C c l 2,0.04$ for Adgre1 and 0.05 for $C d 68$, respectively. e, Same as b but sections were stained with F4/80-targeting antibodies. The number of F4/80+ cells was counted in three to four random fields ( $n=4$ mice per group). Scale bar, $50 \mu \mathrm{m} .{ }^{\star \star \star \star} P<0.0001$. f, mRNA levels for genes encoding thermogenic markers in pgWAT from $C k b^{\text {Adipoq-Cre }}$ and $C k b^{f l / f l}$ mice $(n=6-11$ mice). $\mathbf{g}$, Male mice with a brown adipocyte-specific deletion in $C k b$ expression ( $C k b^{\text {Ucp } 1-C r e E R T 2}$ ) and control littermates ( $C k b^{f l / f l}$ ) were fed a HFD for 16 weeks. Expression of $C k b$ and inflammatory genes was determined in pgWAT ( $n=5-6$ mice per group). Data were analysed by Student's two-sided $t$-test. Data are shown as mean \pm s.e.m

regular basis (every 3 months) and followed the guidelines of the Federation of European Laboratory Animal Science Associations. All experimental procedures were approved by the Stockholm North Animal Ethical Committee (ethical permit N38/15), and special care was taken to minimize animal suffering and to reduce the number of animals used. For HFD experiments, five- to six week-old mice were fed either with CD (4\% kcal from fat, R34; Lantmännen) or HFD (60\% kcal from fat, D12492i, Research Diets) for five weeks. For phosphocreatine injection experiments, mice were fed a standard rodent CD ( $4 \% \mathrm{kcal}$ from fat, R34; Lantmännen). At 11 weeks of age, mice with similar body weights were randomized ( $n=8$ per group) to receive daily intraperitoneal injection of phosphocreatine ( $3 \mathrm{mg} \mathrm{g}^{-1}$ body weight) or PBS ( $20 \mathrm{ml} \mathrm{kg}^{-1}$ body weight) for seven days. This time span was based on the regulations of the animal ethical committee. Four hours before the sacrifice, body weight was measured. Animals were euthanized under general anaesthesia by avertin injection and the wet weight of each dissected tissue was measured. WAT was obtained from the perigonadal (pgWAT) and inguinal (iWAT) depots which were weighed. Samples for qPCR, metabolite and protein analyses were snap-frozen in liquid nitrogen immediately after the wet weight was determined. One part of the fresh tissue samples was fixed in $4 \%$ formalin ( $\mathrm{pH} 7.0$ ) and used for immunofluorescence analyses as described below. The generation, housing and diet intervention of $C k b^{\text {AdipoQ-Cre }}, C k b^{\text {UcP1-CreERT2 }}$ and $C k b^{f / / l}$ animals has been described ${ }^{17}$.

Metabolic evaluations in mice. Glucose tolerance in CD-/HFD-fed animals was determined two days before sacrifice. In brief, glucose $\left(1 \mathrm{~g} \mathrm{~kg}^{-1}\right.$ body weight) was administered by intraperitoneal injection in mice fasted for $4 \mathrm{~h}$. Blood was sampled through the tail vein to measure glucose (OneTouch Ultra 2 glucose meter; LifeScan). Plasma insulin levels were measured by a commercial ELISA kit (Crystal Chem) according to the manufacturer's instructions.

Immunofluorescence analyses. WAT samples were fixed in $4 \%$ paraformaldehyde (PFA) for $24 \mathrm{~h}$, embedded in paraffin and then sectioned $(5 \mu \mathrm{m})$ and stained with hematoxylin and eosin (Sigma-Aldrich). Immunofluorescence was performed as described $^{55}$. Slides were incubated overnight with an Alexa-488-coupled anti-F4/80 (1:50) or anti-CKB (1:100) antibody. Goat anti-Rabbit Rhodamine Red-X (1:500) was used a as a secondary antibody in the CKB experiments. Lens culinaris agglutinin (1:500) and Hoescht (1:500) were applied for $20 \mathrm{~min}$ to counterstain plasma membranes and nuclei, respectively. For each section, the total number of $\mathrm{F} 4 / 80$ positive cells were counted in three to four random $\times 10$ fields using a Axio Observer.Z1 inverted fluorescence microscope (Zeiss) and the AxioVision software.

Adipocyte progenitors were stained for DPP4. In brief, cells were seeded on glass coverslips (thickness no. 1.5) and fixed in 4\% PFA for $15 \mathrm{~min}$ at room temperature. Cells were washed twice with PBS, permeabilized using $0.1 \%$ Triton-X100 for $10 \mathrm{~min}$ and blocked for $30 \mathrm{~min}$ in PBS containing 10\% normal goat serum. Subsequently, cells were incubated for $1 \mathrm{~h}$ with an antibody targeting DPP4 in blocking buffer. Cells were washed three times with PBS and incubated with secondary Alexa Fluor-conjugated antibodies for $1 \mathrm{~h}$. Following three washes with PBS, cells were stained with Hoechst (diluted 1:5,000) for $15 \mathrm{~min}$ and mounted in fluorescence mounting medium (Fluoromount Aqueous Mounting Medium, refractive index 1.4). Images were acquired using a CREST V3 confocal system (Crest Optics) mounted on an inverted Nikon Ti2 microscope equipped with a Prime BSIexpress sCMOS camera (pixel size $6.5 \mu \mathrm{m}$ ) from Photometrics. A Nikon $\times 20 / 0.75$ air objective was used to acquire images.

For TOM20 imaging in differentiated adipocytes, cells transfected with either siC or siCKB were seeded on no. 1.5 glass coverslips and fixed in $4 \%$ PFA for $15 \mathrm{~min}$ at room temperature. Staining was performed as described above for adipocyte progenitors, using an antibody targeting TOM20. Images were acquired using a CREST V3 confocal system (Crest Optics) in widefield mode (no spinning disk inserted) mounted on an inverted Nikon Ti2 microscope equipped with a Prime 95B sCMOS camera (pixel size $11 \mu \mathrm{m}$ ) from Photometrics. A Nikon $\times 60 / 1.4$ oil objective was used to acquire images. The $\times 1.5$ tube lens of the Ti2 was inserted to reach Nyquist sampling in $x y$. A $z$ stack was acquired at Nyquist sampling in $z$ and the stack was deconvolved using Nikon NIS element software and the fast deconvolution algorithm. Mitochondrial content in differentiated adipocytes was 
further evaluated by measurement of fluorescence intensity in in vitro adipocytes using MitoTracker Deep Red FM according to manufacturer's instructions.

Cell culture. Cultures of in vitro differentiated human adipocytes were established and differentiated as described ${ }^{19}$. In brief, cells were obtained from abdominal subcutaneous WAT of one male (16 years, BMI $24 \mathrm{~kg} \mathrm{~m}^{-2}$ ) and one female (39 years, BMI $23.6 \mathrm{~kg} \mathrm{~m}^{-2}$ ) donor.

RNAi experiments and incubations with chemical compounds. Short interfering oligonucleotides (siRNAs) were introduced by electroporation using a Neon Transfection System (1,300 V, 20 ms, 2 pulses) $100 \mu l$ Kit (Invitrogen) in human in vitro differentiated adipocytes at day eight of differentiation. All transfections were performed using a final concentration of $20 \mathrm{nmoll}^{-1}$ siRNA oligonucleotides and were compared with non-silencing control RNAi. In the indicated experiments, at day 13 post-adipogenic induction, human in vitro differentiated adipocytes were treated with phosphocreatine $\left(7.5-30 \mathrm{mmoll}^{-1}\right)$, creatine $(7.5-$ $\left.30 \mathrm{mmoll}^{-1}\right)$, oligomycin $\left(1 \mu \mathrm{moll}^{-1}\right), 2$-deoxy-D-glucose $\left(100 \mu \mathrm{moll}^{-1}\right)$, Bongkrekic acid $\left(1 \mu \mathrm{moll}^{-1}\right)$, UK5099 $\left(10 \mu \mathrm{moll}^{-1}\right)$, ETO $\left(3 \mu \mathrm{moll}^{-1}\right)$, BPTES $\left(10 \mu \mathrm{moll}^{-1}\right)$, $\operatorname{AICAR}\left(250 \mu \mathrm{moll}^{-1}\right)$, Metformin $\left(10 \mathrm{mmoll}^{-1}\right)$, PF-739 $\left(5 \mu \mathrm{moll}^{-1}\right)$ and TNF- $\alpha$ $\left(1.5 \mathrm{ng} \mathrm{ml}^{-1}\right)$ for $24 \mathrm{~h}$.

Cellular triglyceride content and lipid staining. Cellular triglyceride levels were measured on cells plated in 96-well plates, using the Triglyceride Quantification Colorimetric/Fluorometric Kit (Sigma-Aldrich) according to the manufacturer's instructions.

For imaging, cells transfected with either siC or siCKB were fixed in $4 \%$ PFA for $15 \mathrm{~min}$ at room temperature and washed twice with PBS. Cells were stained with BODIPY 493/503 (diluted 1:2,500, ThermoFisher) to stain accumulated lipids and Hoechst (diluted 1:5,000) for $15 \mathrm{~min}$. Cells were then washed four times with PBS and images were acquired using CREST V3 confocal system (Crest Optics) mounted on an inverted Nikon Ti2 microscope equipped with a Prime BSIexpress sCMOS camera (pixel size $6.5 \mu \mathrm{m}$ ) from Photometrics. A Nikon $\times 60 / 1.4$ oil objective was used to acquire images.

Glucose uptake assay. Glucose uptake was determined by measuring radioactivity within cells by liquid scintillation counting. In brief, the day before the assay, insulin was removed from the culture medium. After two washes with PBS, cells were incubated for $50 \mathrm{~min}$ at $37^{\circ} \mathrm{C}$ in insulin-free DMEM (Biochrom AG). Then, $125 \mu \mathrm{moll}^{-1} 2$-deoxy-D-glucose and $0.4 \mu \mathrm{Ci} 2$-deoxy$\left[1-{ }^{3} \mathrm{H}\right]$-D-glucose per well were added for $10 \mathrm{~min}$. Subsequently, the cells were washed three times with cold PBS and lysed in $0.1 \% \mathrm{SDS} / \mathrm{H}_{2} \mathrm{O}$. One part of the lysate was saved for determination of protein concentrations using Pierce BCA Protein determination kit (ThermoFisher). The rest of the lysate was transferred to cuvettes containing scintillation fluid and counts per minute was recorded using a Liquid Scintillation Analyser (Tri-Carb 4910 TR, PerkinElmer) and Optiphase Hisafe 3 (PerkinElmer) as scintillation fluid. Data were normalized to protein concentration

Seahorse assays and CyQUANT analysis. OCRs were measured with an XF96 Seahorse Extracellular Flux Analyzer (Agilent) using Cell Mito Stress Test kits. For measurements of basal OCR, cells were incubated in medium supplemented with $1 \mathrm{mmoll}^{-1}$ pyruvate, $2 \mathrm{mmoll}^{-1}$ glutamine and $10 \mathrm{mmoll}^{-1}$ glucose. To assess maximal respiration, FCCP (uncoupling agent) was titrated from $1-2 \mu \mathrm{moll}^{-1}$. Then, Mito Stress assays were performed by sequential addition of $1.5 \mu \mathrm{moll}^{-1}$ oligomycin (inhibitor of ATP synthesis), $1.5 \mu \mathrm{moll}^{-1} \mathrm{FCCP}$ and $0.5 \mu \mathrm{moll}^{-1}$ rotenone/ antimycin A (inhibitors of complex I and complex III of the respiratory chain, respectively). Mitochondrial ATP production was determined by subtracting the oligomycin-depressed OCR from basal OCR. Mitochondrial fuel stress tests were performed using the XF96 fuel kit after acute injection of UK5099 or BPTES/ ETO. Seahorse data were normalized using ThermoFisher Scientific's fluorescent CyQUANT Kit (ThermoFisher) according to manufacturer's instructions. Immediately after Seahorse analysis, the cells were incubated with the CyQUANT reagent and fluorescence was measured. For estimation of the basal and maximal respiration, the mean non-mitochondrial respiration was subtracted from the mean values of basal and maximal respiration.

Determination of CCL2 and adiponectin secretion and plasma high-sensitivity C-reactive protein. For analyses of condtioned media of in vitro differentiated human adipocytes, samples were collected at day 14 of differentiation and secretion of CCL2 as well as adiponectin was determined by ELISA according to the manufacturer's instructions.

ATP/ADP analyses. Measures of cellular ATP and ADP levels were performed in cell lysates using the ADP/ATP Ratio Assay Kit (Sigma-Aldrich) according to the manufacturer's instructions.

Creatine kinase activity assay. Creatine kinase activity was determined with the Creatine Kinase Assay Kit (Abnova) in tissue/cell lysates according to the manufacturer's specifications.
RNA isolation, complementary DNA synthesis and real-time qPCR. Total RNA was extracted from intact human or murine WAT and cell cultures as described ${ }^{19}$. The concentration, purity and quality of the RNA were measured using Nanodrop 2000 (ThermoFisher) and Agilent 2100 Bioanalyzer (Agilent). Total RNA was reverse transcribed with iScript complementary DNA synthesis kits (BioRad). Assessments of mRNA levels were performed using TaqMan (Applied Biosystems) or SYBR-green (BioRad) assays and relative expression was calculated with the comparative Ct-method, that is, $2^{\Delta \mathrm{C} \text {-target gene }} / 2^{\Delta \mathrm{Ct} \text {-reference gene }}$. All primers and kits, including house-keeping genes, are listed in Supplementary Table 2.

Transcriptomic array. Biotinylated DNA targets were prepared from $150 \mathrm{ng}$ total RNA using the GeneChip WT Plus Reagent Kit according to the manufacturer's instructions. Hybridization, washing and staining was carried out on Clariom S Human arrays, using Affymetrix GeneChip Fluidics Station 450 according to the manufacturer's protocol. The fluorescent intensities were determined with Affymetrix GeneChip Scanner 3000 7G. Data were analysed with packages available from Bioconductor (http://www.bioconductor.org). Normalization and calculation of gene expression was performed with the Robust Multichip Average expression measure using the oligo package ${ }^{56}$. A non-specific filter was first applied to include genes with $\log _{2}$ expression signal $>5$ in at least $50 \%$ of all samples. Principal component analysis was then performed using the FactomineR package and Limma package ${ }^{57}$ was used to identify the differentially expressed genes that were ranked using $t$-statistics in the gene set enrichment analyses (GSEA) ${ }^{58}$. To identify genes/pathways affected by altered CK-B mRNA/protein levels in human adipocytes, GSEA was performed using clusterProfiler package ${ }^{59}$ with Hallmark gene sets from MsigDB (http://www.gsea-msigdb.org/gsea/msigdb/index.jsp). GSEA of genes correlating with $C K B$ expression in human WAT was performed using the available transcriptomics data from cohort 2 (ref. ${ }^{21}$ ). In brief, probesets were merged at the gene level using collapseRows function (default setting) in WGCNA package ${ }^{60}$. Genes were then ranked based on their association with $C K B$ using Spearman's rank correlation coefficient $(\rho)$ before performing GSEA.

Western blot analyses. Western blots were performed as described previously ${ }^{19}$. All antibodies are listed in Supplementary Table 2. For blots where the proteins of interest run at the same size, lysates were subdivided in equal amounts and loaded on two separate gels.

Isolation of mitochondria and cytoplasm from in vitro differentiated human adipocytes. in vitro differentiated human adipocytes were homogenized in ice-cold isolation buffer $\left(250 \mathrm{mmoll}^{-1}\right.$ sucrose buffer, $20 \mathrm{mmoll}^{-1}$ Tris- $\mathrm{HCl}$ ( $\left.\mathrm{pH} 7.4\right)$, $1 \mathrm{mmoll}^{-1}$ EDTA (pH 8), 2\% BSA) using a $27 \mathrm{G}$ syringe. The lysate was centrifuged at $1,000 \mathrm{~g}$ for $15 \mathrm{~min}$ to remove the nuclei. The supernatant was centrifuged at $8,500 \mathrm{~g}$ for $15 \mathrm{~min}$ to isolate mitochondria and the mitochondrial pellet was resuspended in TES buffer $\left(100 \mathrm{mmoll}^{-1} \mathrm{KCl}, 20 \mathrm{mmoll}^{-1} \mathrm{TES}, 1 \mathrm{mmoll}^{-1}\right.$ EGTA, 0.6\% BSA (pH 7.2)). After mitochondrial isolation, the supernatant was centrifuged for $2 \mathrm{~h}$ at $20,000 \mathrm{~g}$ to remove the membranes and separate the lipid droplets. The remaining supernatant was used as cytoplasmic fraction.

Targeted metabolite analyses in cells and murine tissues. Metabolites were profiled at the Swedish Metabolomics Centre, the Helmholtz Zentrum and at McGill University. For the latter, the analyses were performed on WAT from $C k b^{\text {Adipoq-Cre }}$ and $C k b^{f l f l}$ and the methods have been described ${ }^{17}$. For analyses performed at the Swedish Metabolomics Centre, human in vitro differentiated adipocytes were lysed at day 14 post-induction of differentiation in $1 \mathrm{ml}$ of $90 \%$ methanol (diluted $\mathrm{H}_{2} \mathrm{O}$ ) containing $0.5 \mathrm{\mu moll}^{-1}$ creatine- $\mathrm{D}_{3}$ as an internal standard, shaken with metal beads at $30 \mathrm{kHz}$ for $3 \mathrm{~min}$ and centrifuged at $14,000 \mathrm{~g}$ for $10 \mathrm{~min}$. The supernatants were divided in two aliquots for both liquid (LC) and gas (GC) chromatography analyses. Phosphocreatine and creatine were analysed using LC-tandem mass spectrometry. The separation was achieved by using hydrophilic interaction chromatography (iHILIC-(P) Classic, PEEK, $150 \mathrm{~mm} \times 2.1 \mathrm{~mm}$, $5 \mu \mathrm{m}$, HILICON). The LC-tandem mass spectrometry system consisted of an Agilent 1290 UPLC connected to an Agilent 6490 triple quadrupole tandem mass spectrometer (Agilent). Analytes were ionized in electrospray source operated in the positive (creatine) and negative (phosphocreatine) mode. The analyses were performed in multiple reaction monitoring mode. Glycolytic intermediates were analysed by GC-MS. Before the evaporation of the supernatant, it was spiked with $1.05 \mathrm{ng}$ of each GC-MS internal standards. Derivatization was performed as described ${ }^{61}$. The GC-MS system consisted of an Agilent 7693 autosampler, an Agilent 7890A gas chromatograph and an Agilent 7000C QQQ mass spectrometer. Ions were generated by a $70 \mathrm{eV}$ electron beam and analysed in dynamic multiple reaction monitoring mode. Data were processed and analysed using MassHunter Qualitative Analysis, Quantitative Analysis (QqQ; Agilent) and Excel (Microsoft) software. For analyses performed at the Helmoltz Zentrum Munchen, metabolites were extracted from cell pellets of in vitro differentiated adipocytes using $1 \mathrm{ml}$ of cold $\mathrm{CHCl}_{3} / \mathrm{MeOH} / \mathrm{H}_{2} \mathrm{O}(1 / 3 / 1, \mathrm{v} / \mathrm{v} / \mathrm{v})$. After suspension in solvent, cells were transferred to $2 \mathrm{ml} \mathrm{MN}$ Bead Tubes Type A (Macherey-Nagel) and lysed using a Precellys Bead Beating system with an additional Cryolys cooling module (Bertin Instruments). After lysis, samples were incubated for $10 \mathrm{~min}$ in an ice-cold ultrasonic bath followed by centrifugation at $4^{\circ} \mathrm{C}$ for $15 \mathrm{~min}$. The supernatant was 
transferred to a fresh reaction tube and evaporated to dryness using a centrifugal evaporator. Proteins were extracted from the residue cell debris pellet and quantified using a BCA Kit (Sigma-Aldrich). Metabolite profiling was performed using a Sciex Exion AD LC coupled to a Sciex X500R Q-ToF-MS (Sciex). Separation was achieved on an Agilent InfinityLab Poroshell 120 HILIC-Z column (2.1 mm $\times 150 \mathrm{~mm}, 2.7 \mu \mathrm{m}$ particle size, PEEK-lined) (Agilent Technologies). Analysis conditions were similar to Hsiao et al. ${ }^{62}$. Dried samples were re-dissolved in $50 \mu \mathrm{CHCl}_{3} / \mathrm{MeOH} / \mathrm{H}_{2} \mathrm{O}(1 / 3 / 1, \mathrm{v} / \mathrm{v} / \mathrm{v})$ and $40 \mu \mathrm{l}$ were transferred to an autosampler vial and $10 \mu \mathrm{l}$ to a pooled quality control sample. These samples were used for conditioning of the column and were also injected every ten samples. Data analysis was performed in Sciex OS (Sciex). Peaks for creatine $\left(\mathrm{C}_{4} \mathrm{H}_{9} \mathrm{~N}_{3} \mathrm{O}_{2}\right.$, $\left.[\mathrm{M}+\mathrm{H}]^{+}, 10.42 \mathrm{~min}\right)$ and phosphocreatine $\left(\mathrm{C}_{4} \mathrm{H}_{10} \mathrm{~N}_{3} \mathrm{O}_{5} \mathrm{P},[\mathrm{M}+\mathrm{H}]^{+}, 13.63 \mathrm{~min}\right)$ were integrated with an extracted ion chromatogram (width of $0.02 \mathrm{Da}$ and a Gaussian smooth width of three points using the MQ4 peak picking algorithm. Identity of peaks was confirmed using authentic standards of all substances and comparison against reference spectra and peak areas were normalized according to the protein content of the respective sample.

Statistics. Data are reported as mean \pm s.e.m. unless otherwise stated. Results were compared by one- or two-tailed Student's $t$-test or one- or two-way analysis of variance (ANOVA) with Tukey's post-hoc test. For correlation analyses in clinical cohorts, simple and multiple regression analyses (with BMI included as independent regressor) were used. The number of independent experiments and relevant statistical methods for each panel are detailed in the figure legends. Statistical analyses of cell and animal data were performed using Prism (GraphPad Software), analyses of clinical data were done using JMP (v.15.1, SAS) and bioinformatic analyses were performed with R v.4.1.1.

Reporting Summary. Further information on research design is available in the Nature Research Reporting Summary linked to this article.

\section{Data availability}

Microarray data generated or retrospectively analysed in this study are publicly available in the NCBI Gene Expression Omnibus repository under the accession numbers GSE25401 (transcriptomics of cohort 2), GSE59034 (transcriptomics of cohort 3), GSE100795 (transcriptomics of different resident cells of human WAT) and GSE192361 (transcriptomics of siCKB-versus siC-transfected cells). Metabolomics data from cohort 1 are provided in ref. ${ }^{19}$. RNA sequencing data at multiple time points during adipocyte differentiation are available from these cells within the FANTOM5 project $^{63}$ and was used to extract information on genes within the phosphocreatine/creatine pathway presented in Fig. 2b. Source data are provided with this paper. Additional data that support the findings of this study are available from the corresponding authors upon reasonable request.

Received: 30 April 2021; Accepted: 5 January 2022; Published online: 14 February 2022

\section{References}

1. Rosen, E. D. \& Spiegelman, B. M. What we talk about when we talk about fat. Cell 156, 20-44 (2014).

2. Mejhert, N. \& Ryden, M. Novel aspects on the role of white adipose tissue in type 2 diabetes. Curr. Opin. Pharmacol. 55, 47-52 (2020).

3. Hotamisligil, G. S. Inflammation, metaflammation and immunometabolic disorders. Nature 542, 177-185 (2017).

4. Crewe, C., An, Y. A. \& Scherer, P. E. The ominous triad of adipose tissue dysfunction: inflammation, fibrosis, and impaired angiogenesis. J. Clin. Invest. 127, 74-82 (2017).

5. Reilly, S. M. \& Saltiel, A. R. Adapting to obesity with adipose tissue inflammation. Nat. Rev. Endocrinol. 13, 633-643 (2017).

6. Hotamisligil, G. S., Shargill, N. S. \& Spiegelman, B. M. Adipose expression of tumor necrosis factor-alpha: direct role in obesity-linked insulin resistance. Science 259, 87-91 (1993).

7. Berger, S. L. \& Sassone-Corsi, P. Metabolic signaling to chromatin. Cold Spring Harb. Perspect. Biol 8, A019463 (2016).

8. Husted, A. S., Trauelsen, M., Rudenko, O., Hjorth, S. A. \& Schwartz, T. W. GPCR-Mediated Signaling of Metabolites. Cell Metab. 25, 777-796 (2017)

9. Liu, G. Y. \& Sabatini, D. M. mTOR at the nexus of nutrition, growth, ageing and disease. Nat. Rev. Mol. Cell Biol. 21, 183-203 (2020).

10. Ryan, D. G. et al. Coupling Krebs cycle metabolites to signalling in immunity and cancer. Nat. Metab. 1, 16-33 (2019).

11. Lecoutre, $\mathrm{S}$. et al. Glutamine metabolism in adipocytes: a bona fide epigenetic modulator of inflammation. Adipocyte 9, 620-625 (2020).

12. Buck, M. D., Sowell, R. T., Kaech, S. M. \& Pearce, E. L. Metabolic instruction of immunity. Cell 169, 570-586 (2017).

13. Kucera, J. et al. Human white adipose tissue metabolome: current perspective. Obesity (Silver Spring) 26, 1870-1878 (2018).

14. Kazak, L. et al. Ablation of adipocyte creatine transport impairs thermogenesis and causes diet-induced obesity. Nat. Metab. 1, 360-370 (2019).
15. Kazak, L. et al. Genetic depletion of adipocyte creatine metabolism inhibits diet-induced thermogenesis and drives obesity. Cell Metab. 26, 693 (2017).

16. Kazak, L. \& Cohen, P. Creatine metabolism: energy homeostasis, immunity and cancer biology. Nat. Rev. Endocrinol. 16, 421-436 (2020).

17. Rahbani, J. F. et al. Creatine kinase B controls futile creatine cycling in thermogenic fat. Nature 590, 480-485 (2021).

18. Ji, L. et al. Slc6a8-mediated creatine uptake and accumulation reprogram macrophage polarization via regulating cytokine responses. Immunity 51, 272-284.e277 (2019).

19. Petrus, P. et al. Glutamine links obesity to inflammation in human white adipose tissue.Cell Metab. 31, 375-390.e311 (2020).

20. Maguire, O. A. et al. Creatine-mediated crosstalk between adipocytes and cancer cells regulates obesity-driven breast cancer. Cell Metab. 33, 499-512. e496 (2021)

21. Arner, E. et al. Adipose tissue microRNAs as regulators of CCL2 production in human obesity. Diabetes 61, 1986-1993 (2012).

22. Petrus, P. et al. Transforming growth factor-beta 3 regulates adipocyte number in subcutaneous white adipose tissue. Cell Rep. 25, 551-560.e555 (2018).

23. Acosta, J. R. et al. Single cell transcriptomics suggest that human adipocyte progenitor cells constitute a homogeneous cell population. Stem Cell Res. Ther. 8, 250 (2017).

24. Carthew, R. W. Gene regulation and cellular metabolism: an essential partnership. Trends Genet. 37, 389-400 (2021).

25. Kanda, H. et al. MCP-1 contributes to macrophage infiltration into adipose tissue, insulin resistance, and hepatic steatosis in obesity. J. Clin. Invest. 116 1494-1505 (2006).

26. Kim, C. S. et al. Circulating levels of MCP-1 and IL- 8 are elevated in human obese subjects and associated with obesity-related parameters. Int. J. Obes. 30, 1347-1355 (2006).

27. Kamei, N. et al. Overexpression of monocyte chemoattractant protein-1 in adipose tissues causes macrophage recruitment and insulin resistance. J. Biol. Chem. 281, 26602-26614 (2006).

28. Weisberg, S. P. et al. CCR2 modulates inflammatory and metabolic effects of high-fat feeding. J. Clin. Invest. 116, 115-124 (2006).

29. Kazak, L. et al. A creatine-driven substrate cycle enhances energy expenditure and thermogenesis in beige fat. Cell 163, 643-655 (2015).

30. Boudina, S. \& Graham, T. E. Mitochondrial function/dysfunction in white adipose tissue. Exp. Physiol. 99, 1168-1178 (2014).

31. Missiroli, S. et al. The role of mitochondria in inflammation: from cancer to neurodegenerative disorders. J. Clin. Med. 9, 740 (2020).

32. Liu, T. F. et al. Fueling the flame: bioenergy couples metabolism and inflammation. J. Leukoc. Biol. 92, 499-507 (2012).

33. Martinez-Reyes, I. \& Chandel, N. S. Mitochondrial TCA cycle metabolites control physiology and disease. Nat. Commun. 11, 102 (2020).

34. O’Neill, L. A. \& Hardie, D. G. Metabolism of inflammation limited by AMPK and pseudo-starvation. Nature 493, 346-355 (2013).

35. Salminen, A., Hyttinen, J. M. \& Kaarniranta, K. AMP-activated protein kinase inhibits NF-kappaB signaling and inflammation: impact on healthspan and lifespan. J. Mol. Med. 89, 667-676 (2011).

36. Ping, D., Jones, P. L. \& Boss, J. M. TNF regulates the in vivo occupancy of both distal and proximal regulatory regions of the MCP-1/JE gene. Immunity 4, 455-469 (1996).

37. Lee, T. H., Lee, G. W., Ziff, E. B. \& Vilcek, J. Isolation and characterization of eight tumor necrosis factor-induced gene sequences from human fibroblasts. Mol. Cell. Biol. 10, 1982-1988 (1990).

38. Sarvari, A. K. et al. Plasticity of epididymal adipose tissue in response to diet-induced obesity at single-nucleus resolution. Cell Metab. 33, 437-453. e435 (2021).

39. Joncquel-Chevalier Curt, M. et al. Creatine biosynthesis and transport in health and disease. Biochimie 119, 146-165 (2015).

40. O’Neill, L. A. \& Pearce, E. J. Immunometabolism governs dendritic cell and macrophage function. J. Exp. Med. 213, 15-23 (2016).

41. Keuper, M. On the role of macrophages in the control of adipocyte energy metabolism. Endocr. Connect. 8, R105-R121 (2019).

42. Bijland, S., Mancini, S. J. \& Salt, I. P. Role of AMP-activated protein kinase in adipose tissue metabolism and inflammation. Clin. Sci. 124, 491-507 (2013).

43. Zhao, P. et al. TBK1 at the crossroads of inflammation and energy homeostasis in adipose tissue. Cell 172, 731-743.e712 (2018).

44. Martin, T. L. et al. Diet-induced obesity alters AMP kinase activity in hypothalamus and skeletal muscle. J. Biol. Chem. 281, 18933-18941 (2006).

45. Viollet, B. et al. AMPK inhibition in health and disease. Crit. Rev. Biochem. Mol. Biol. 45, 276-295 (2010).

46. Xu, X. J. et al. Insulin sensitive and resistant obesity in humans: AMPK activity, oxidative stress, and depot-specific changes in gene expression in adipose tissue. J. Lipid Res. 53, 792-801 (2012).

47. Day, E. A., Ford, R. J. \& Steinberg, G. R. AMPK as a therapeutic target for treating metabolic diseases. Trends Endocrinol. Metab. 28, 545-560 (2017) 
48. Salt, I. P. \& Palmer, T. M. Exploiting the anti-inflammatory effects of AMP-activated protein kinase activation. Expert Opin. Investig. Drugs 21, 1155-1167 (2012).

49. Ponticos, M. et al. Dual regulation of the AMP-activated protein kinase provides a novel mechanism for the control of creatine kinase in skeletal muscle. EMBO J. 17, 1688-1699 (1998).

50. Wernstedt Asterholm, I. et al. Adipocyte inflammation is essential for healthy adipose tissue expansion and remodeling. Cell Metab 20, 103-118 (2014).

51. Zhu, Q. et al. Suppressing adipocyte inflammation promotes insulin resistance in mice. Mol. Metab. 39, 101010 (2020).

52. Papalazarou, V. et al. The creatine-phosphagen system is mechanoresponsive in pancreatic adenocarcinoma and fuels invasion and metastasis. Nat. Metab. 2, 62-80 (2020).

53. Mekhfi, $H$. et al. Creatine kinase is the main target of reactive oxygen species in cardiac myofibrils. Circ. Res. 78, 1016-1027 (1996).

54. Jabs, C. M., Ferrell, W. J. \& Robb, H. J. Microdetermination of plasma ATP and creatine phosphate concentrations with a luminescence biometer. Clin. Chem. 23, 2254-2257 (1977)

55. Berry, R. et al. Imaging of adipose tissue. Methods Enzymol. 537, 47-73 (2014).

56. Carvalho, B. S. \& Irizarry, R. A. A framework for oligonucleotide microarray preprocessing. Bioinformatics 26, 2363-2367 (2010).

57. Ritchie, M. E. et al. limma powers differential expression analyses for RNA-sequencing and microarray studies. Nucleic Acids Res. 43, e47 (2015).

58. Subramanian, A. et al. Gene set enrichment analysis: a knowledge-based approach for interpreting genome-wide expression profiles. Proc. Natl Acad. Sci. USA 102, 15545-15550 (2005).

59. Yu, G., Wang, L. G., Han, Y. \& He, Q. Y. clusterProfiler: an R package for comparing biological themes among gene clusters. OMICS $\mathbf{1 6}$ 284-287 (2012).

60. Langfelder, P. \& Horvath, S. WGCNA: an R package for weighted correlation network analysis. BMC Bioinform. 9, 559 (2008).

61. Gullberg, J., Jonsson, P., Nordstrom, A., Sjostrom, M. \& Moritz, T. Design of experiments: an efficient strategy to identify factors influencing extraction and derivatization of Arabidopsis thaliana samples in metabolomic studies with gas chromatography/mass spectrometry. Anal. Biochem. 331, 283 (2004).

62. Hsiao, J. J., Potter, O. G., Chu, T. W. \& Yin, H. Improved LC/MS methods for the analysis of metal-sensitive analytes using medronic acid as a mobile phase additive. Anal. Chem. 90, 9457-9464 (2018).

63. Ehrlund, A. et al. Transcriptional dynamics during human adipogenesis and its link to adipose morphology and distribution. Diabetes 66, 218-230 (2017).

\section{Acknowledgements}

We thank L. Kazak, B. Samborska and J. Rahbani (McGill University, Montreal) for providing samples from the adipocyte-specific $C k b$ knockout animals and for constructive comments on the manuscript together with B. M. Spiegelman (Dana-Farber Cancer Institute, Boston). This work was supported by grants from Margareta af Uggla's foundation (M.R.), the Swedish Research Council (M.R. and N.M.), ERC-SyG SPHERES (856404 to M.R.), the NovoNordisk Foundation (including the Tripartite Immuno-metabolism Consortium grant number NNF15CC0018486, the MSAM consortium NNF15SA0018346 and the MeRIAD consortium grant number 0064142 to M.R. and NNF20OC0061149 to N.M.), Knut and Alice Wallenbergs Foundation (A.K., J.R.Z, M.R. and T.M., including Wallenberg Clinical Scholar to M.R.), CIMED (N.M. and
M.R.), the Swedish Diabetes Foundation (M.R.), the Stockholm County Council (M.R.) and the Strategic Research Programme in Diabetes at Karolinska Institutet (A.K., J.R.Z., M.R. and H.G.). S.M. was supported by the Université Clermont Auvergne, Société Francophone du Diabète and Fondation Bettencourt Schueller. S.F.C. is supported by a Novo Nordisk postdoctoral fellowship run in partnership with Karolinska Institutet. Fluorescent imaging was performed at the Live Cell imaging Core facility/Nikon Center of Excellence, at the Karolinska Institute, supported by grants from the Swedish Research Council, KI infrastructure and Centre for Innovative Medicine.

\section{Author contributions}

S.M., S.L., N.M. and M.R. conceived the idea and wrote the manuscript, which was then read, commented and approved by all authors. M.R. collected all clinical samples. S.M., S.L. D.R.-R., J.R.Z. and A. Krook., performed the HFD and phosphocreatine studies in mice. H.G. performed bioinformatic analyses. A. Kerr., G.R., S.F.-C. and M.C. contributed to in vitro assays. M.O.B., M.A. and R.P.C. contributed to data analyses and interpretation. T.M., M.J., O.H. and M.W. performed the targeted metabolite analyses in human in vitro differentiated adipocytes and murine tissues. S.M. and S.L. put together all source data.

\section{Funding}

Open access funding provided by Karolinska Institute.

\section{Competing interests}

The authors declare no competing interests.

\section{Additional information}

Extended data is available for this paper at https://doi.org/10.1038/s42255-022-00525-9.

Supplementary information The online version contains supplementary material available at https://doi.org/10.1038/s42255-022-00525-9.

Correspondence and requests for materials should be addressed to Niklas Mejhert or Mikael Rydén.

Peer review information Nature Metabolism thanks David Carling and the other anonymous, reviewer(s) for their contribution to the peer review of this work. Primary handling editor: Isabella Samuelson.

Reprints and permissions information is available at www.nature.com/reprints.

Publisher's note Springer Nature remains neutral with regard to jurisdictional claims in published maps and institutional affiliations.

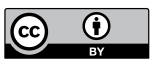

Open Access This article is licensed under a Creative Commons

Attribution 4.0 International License, which permits use, sharing, adaptation, distribution and reproduction in any medium or format, as long as you give appropriate credit to the original author(s) and the source, provide a link to the Creative Commons license, and indicate if changes were made. The images or other third party material in this article are included in the article's Creative Commons license, unless indicated otherwise in a credit line to the material. If material is not included in the article's Creative Commons license and your intended use is not permitted by statutory regulation or exceeds the permitted use, you will need to obtain permission directly from the copyright holder. To view a copy of this license, visit http://creativecommons. org/licenses/by/4.0/.

(c) The Author(s) 2022 
A

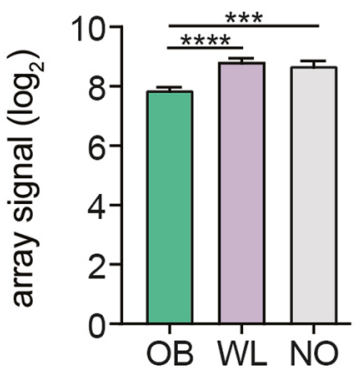

SLC6A8

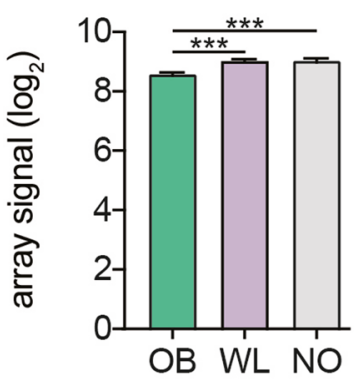

CKM

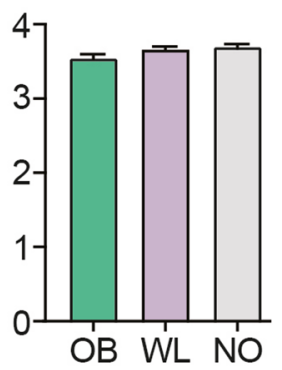

SLC6A6

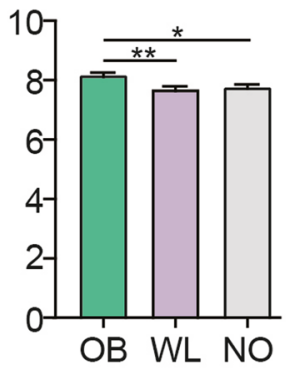

CKMT1

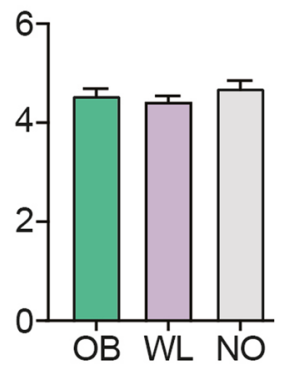

GATM

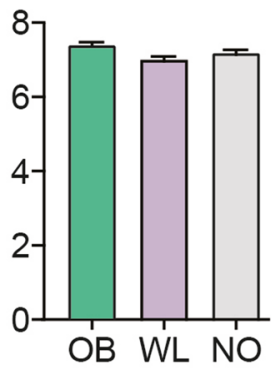

CKMT2

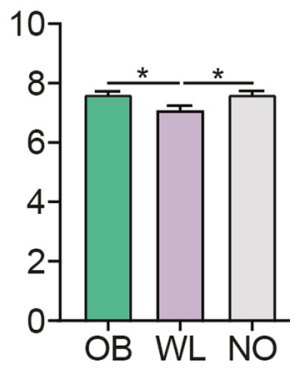

B

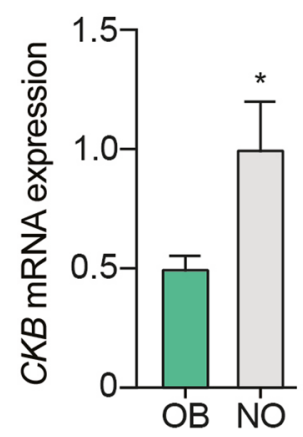

Extended Data Fig. 1 | CKB expression is regulated by obesity in both women and men. a. Expression levels of genes encoding proteins in phosphocreatine/creatine metabolism in cohort 3 consisting of 15 women before (OB) and two years after weight loss (WL) induced by bariatric surgery. The WL group was matched by age and body mass index to a group of 15 never-obese subjects (NO). Data are shown as mean \pm SEM. Data were analyzed by one-way ANOVA. Overall p-values were $<0.0001$ for CKB, SLC6A8 and GAMT, 0.010 for CKMT2 and 0.0023 for SLC6A6. Results from Tukey's post-hoc test are indicated by ${ }^{\star} p<0.05,{ }^{\star \star} p<0.01,{ }^{\star \star \star} p<0.001$ and ${ }^{\star \star \star \star} p<0.0001$, respectively. $\mathbf{b}$. White adipose tissue expression levels of $C K B$ determined by qPCR in samples obtained from 18 obese and 17 non-obese men (cohort 4). Data are shown as mean \pm SEM. ${ }^{*} p=0.021$ by Student's two-sided t-test. 
A
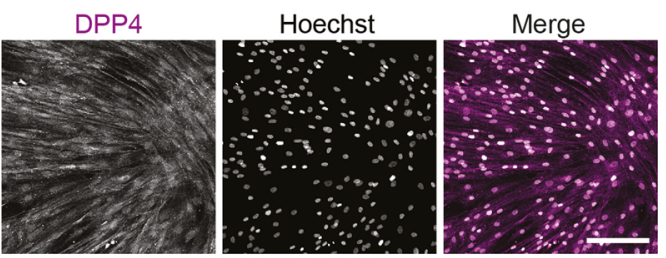

C

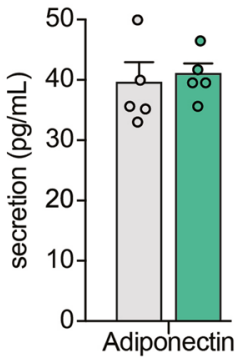

D

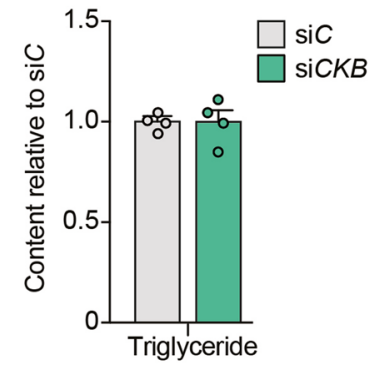

$\mathbf{F}$

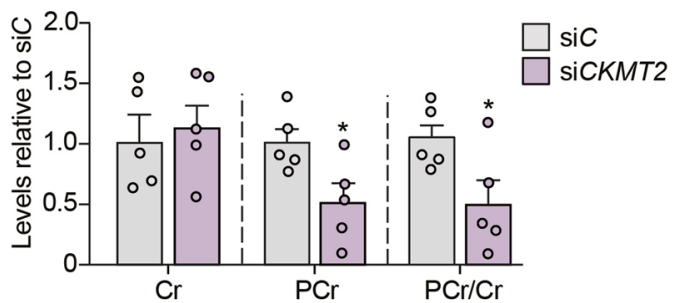

H

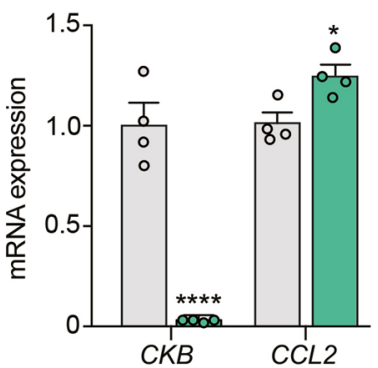

I

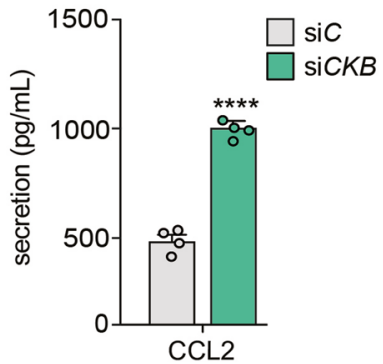

B

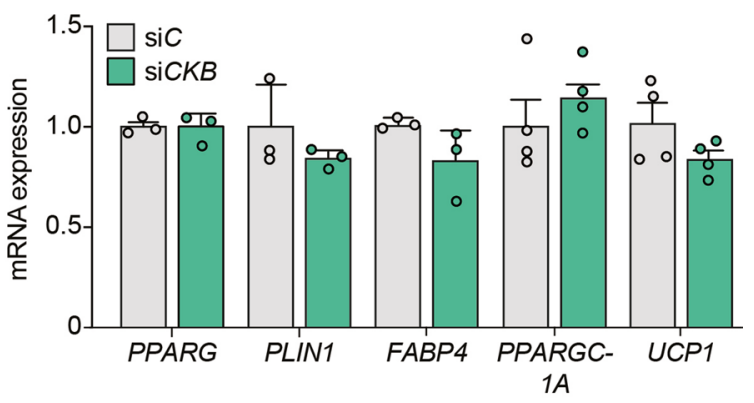

E
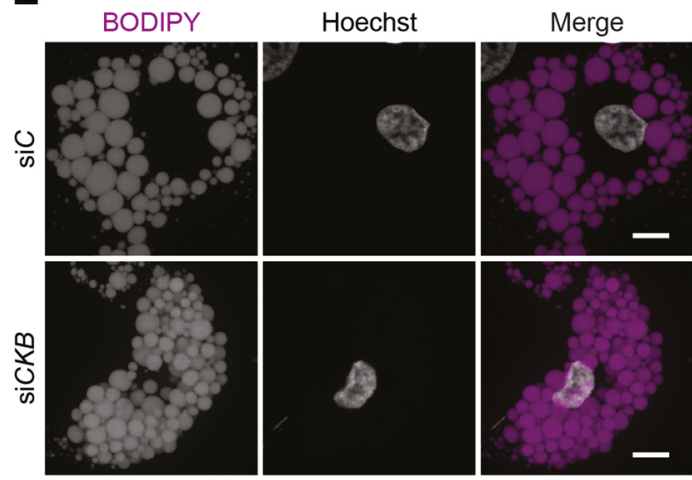

G

Pathways associated with $C K B$ mRNA expression

Epithelial mesenchymal transitionCoagulation Inflammatory response. Allograft rejection

TNFa signaling via NFKB Apical junction KRAS signaling up IL6-JAK-STAT3 signaling Myogenesis

KRAS signaling $\mathrm{dn}$

IL2-STAT5 signaling Complement. Hypoxia Interferon gamma response Peroxisome Adipogenesis

Fatty acid metabolism Oxidative phosphorylation

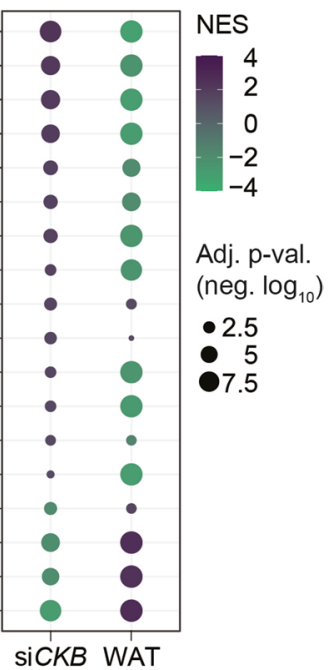

Extended Data Fig. 2 | CKB depletion does not affect adipogenesis. a. Representative immunofluorescence microphotographs of adipocyte progenitors stained with Hoechst and antibodies targeting DPP4. Scale bar $=200 \mu \mathrm{m}$. b-e. In vitro differentiated human adipocytes were transfected with scrambled non-silencing ( $\mathrm{siC}$ ) or CKB-targeting (siCKB) (three-four replicates/condition). Effects on gene expression of adipogenic/thermogenic markers (experiment repeated twice) (B), adiponectin secretion (five replicates/condition) (C), cellular triglyceride levels (four replicates/condition) (D) and lipid droplet morphology (from experiments in panel D) (E) were determined. Scale bar in panel $E=10 \mu \mathrm{m}$. Data in bar charts are shown as mean $\pm \mathrm{SEM}$. f. PCr and $\mathrm{Cr}$ levels in in vitro differentiated human adipocytes transfected with scrambled non-silencing ( $\mathrm{SiC}$ ) or CKMT2-targeting ( $s i C K M T 2)$ oligonucleotides ( $n=5$ replicates/group). Data are shown as mean $\pm S E M$. ${ }^{\star} p=0.024$ for $\mathrm{PCr}$ and 0.045 for PCr/Cr, respectively by Student's two-sided t-test. g. Gene set enrichment analysis (GSEA) of genes correlating with CKB expression in human WAT and regulated by siCKB transfection in human in vitro differentiated adipocytes. Hallmark gene sets from MsigDB were used to calculate the enrichment. Details on the analyses are found in the Methods section. The data are presented as dot plots. The color of the dot indicates the normalized enrichment score (NES) for each pathway. The size of the dot represents the negative $\log _{10}$ adjusted $p$-value for the enrichment of each pathway. $\mathbf{h}$. $C K B$ and $C C L 2$ mRNA levels in in vitro differentiated human adipocytes, derived from a female donor, transfected with $\mathrm{siC}$ ) or $\mathrm{siCKB}$ (four replicates/condition). Data are shown as mean $\pm \mathrm{SEM}$. ${ }^{*} \mathrm{p}=0.024$, ${ }^{\star \star \star \star} p<0.0001$ by Student's two-sided t-test. i. Same experiment as in panel H but displaying CCL2 secretion (four replicates/condition). Data are shown as mean \pm SEM. ${ }^{\star \star \star \star} p<0.0001$ by Student's two-sided t-test. Abbreviations: Adj. $p$-val.=adjusted $p$-value, $\mathrm{Cr}=\mathrm{creatine}, \mathrm{NES}=$ normalized enrichment score, $\mathrm{PCr}=$ phosphocreatine, WAT $=$ white adipose tissue. 
A

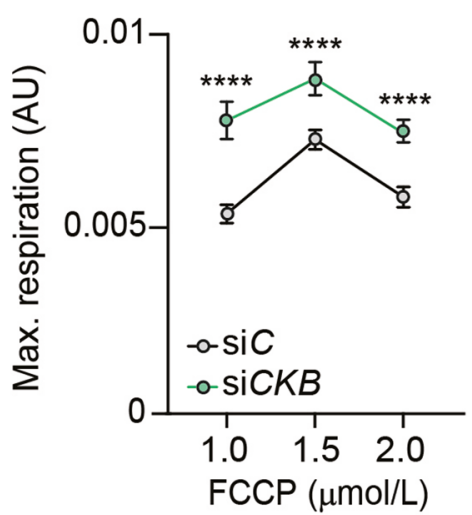

D
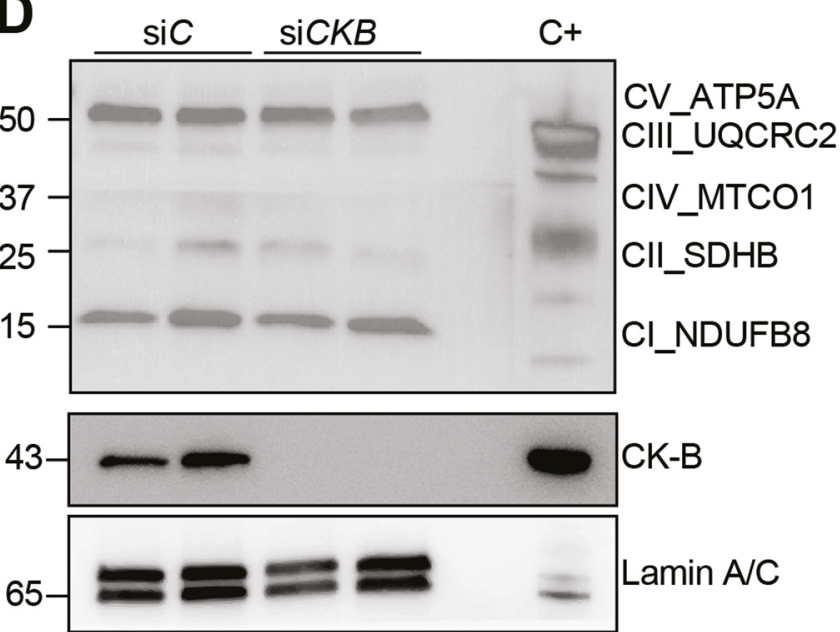

B

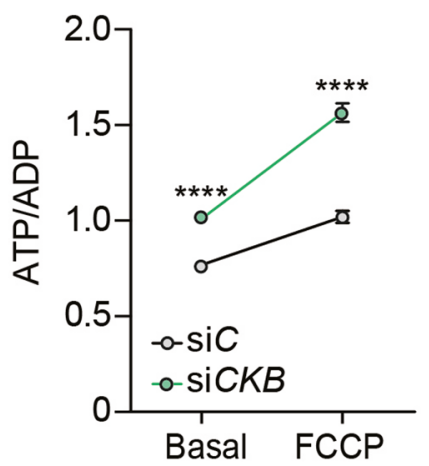

C

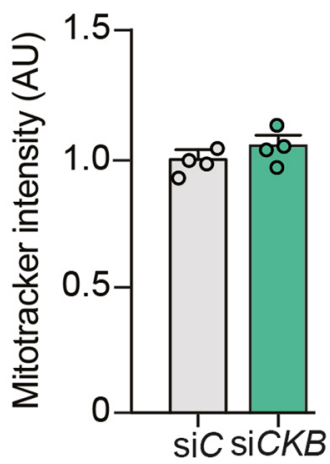

E
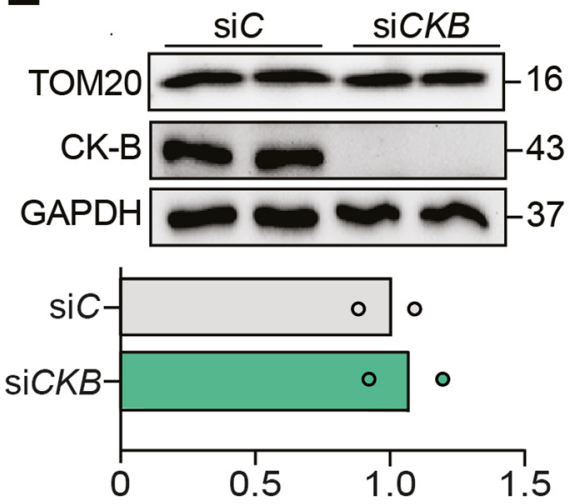

Relative TOM20 protein

$\mathbf{F}$

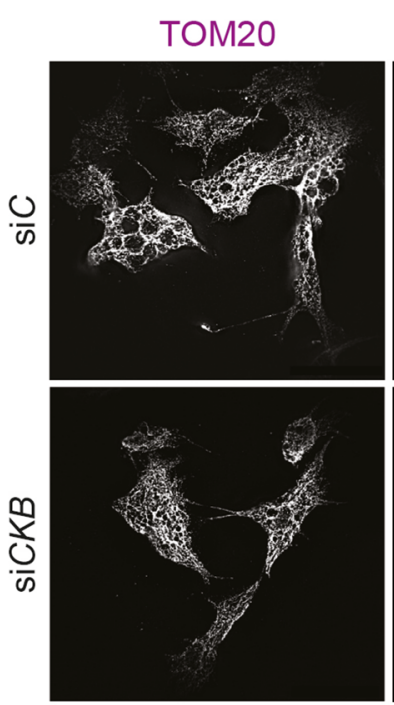

Hoechst

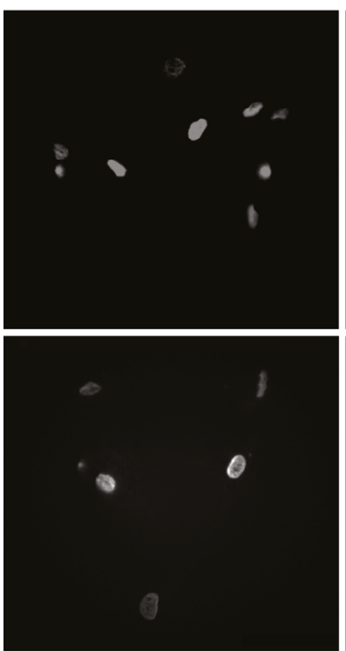

Merge

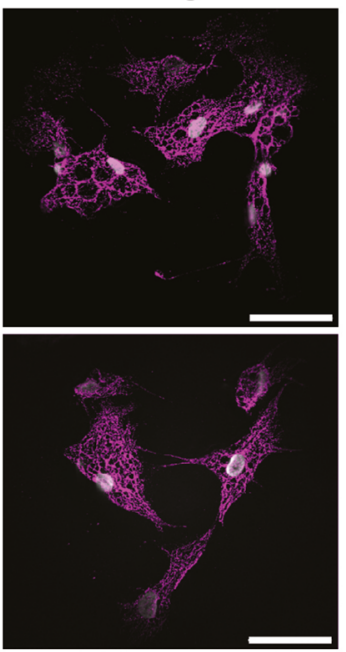

Extended Data Fig. 3 | See next page for caption. 
Extended Data Fig. 3 | CKB depletion does not affect mitochondrial content and morphology. a. Maximal respiration of in vitro differentiated human adipocytes determined by Seahorse Mito stress analysis using 1-2 $\mu \mathrm{mol} / \mathrm{L}$ of FCCP, after transfection of the cells with siC or siCKB (12 replicates/ condition). Data are shown as mean \pm SEM. ${ }^{* \star \star \star} \mathrm{p}<0.0001$ by Student's two-sided $t$-test. $\mathbf{b}$. ATP/ADP levels in in vitro differentiated human adipocytes transfected with siC or siCKB after 1 hour of incubation with $1.5 \mu \mathrm{mol} / \mathrm{L}$ of FCCP (eight replicates per condition). Data are shown as mean \pm SEM. ${ }^{\star \star \star \star} \mathrm{p}<0.0001$ by Student's two-sided t-test. c. Quantification of Mitotracker ${ }^{\mathrm{TM}}$ red fluorescence intensity in in vitro differentiated human adipocytes transfected with siC or siCKB (four replicates/condition, repeated twice). Data are shown as mean $\pm \mathrm{SEM}$. d-e. In vitro differentiated human adipocytes were transfected with siC or siCKB (two replicates per blot). Protein levels of OXPHOS components (D) and TOM2O (mean values) (E) were determined together with CK-B and GAPDH by western blot to estimate mitochondrial abundance. $\mathbf{f}$. Representative immunofluorescence microphotographs of in vitro differentiated adipocytes transfected with $\mathrm{siC}$ or siCKB and stained with antibodies targeting TOM2O and Hoechst. Scale bar $=50 \mu \mathrm{m}$. Abbreviations: ATP5A = Mitochondrial membrane ATP Synthase, AU = arbitrary units, FCCP = Carbonyl cyanide-p-trifluoromethoxyphenylhydrazone, $C+=$ positive control, mitochondrial extract from rat heart supplied with the antibody as a positive control, MTCO1=Mitochondrially Encoded Cytochrome $C$ Oxidase I, NDUFB8 = NADH:Ubiquinone Oxidoreductase Subunit B8, SDHB = Succinate dehydrogenase B, UQCRC2=Cytochrome b-c1 complex subunit 2, mitochondrial. 

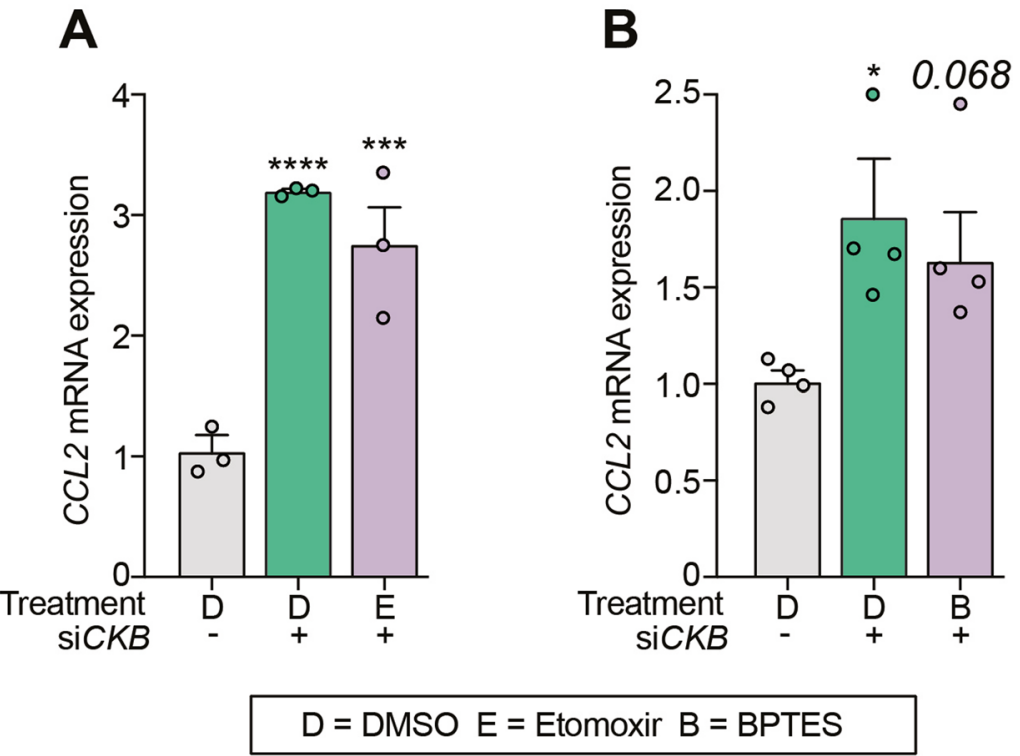

Extended Data Fig. 4 | White adipocyte mitochondrial hyperactivity following CKB silencing is not driven by fatty acid oxidation or glutaminolysis. a-b. CCL2 expression in in vitro differentiated human adipocytes transfected with either siC or siCKB, and treated with $3 \mu$ mol/L Etomoxir (fatty acid oxidation inhibitor) (three replicates/condition) (A) or $10 \mu \mathrm{mol} / \mathrm{L}$ BPTES (glutaminolysis inhibitor) (four replicates per condition) (B). Control cells were treated with DMSO. Data are shown as mean \pm SEM. Overall $p<0.0001$ for panel A and 0.028 for panel B by one-way ANOVA. Results from Tukey's post-hoc test are indicated by ${ }^{\star} p<0.05,{ }^{* \star \star}<0.001$ and ${ }^{* \star \star \star} p<0.0001$, respectively. Abbreviations: BPTES $=$ Bis-2-(5-phenylacetamido-1,3,4-thiadiazol-2-yl)ethyl sulfide, $\mathrm{DMSO}=$ dimethyl sulfoxide. 
A

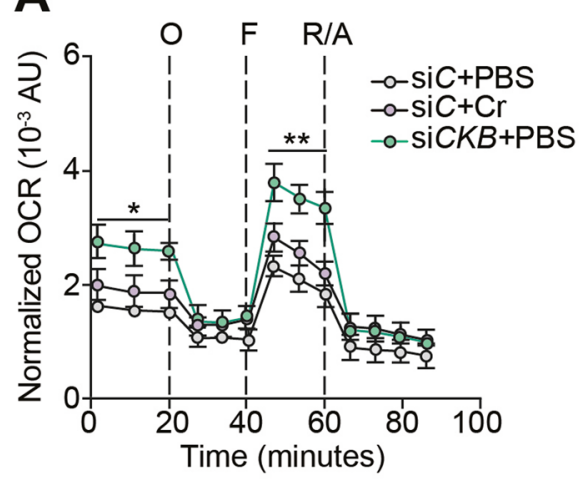

B

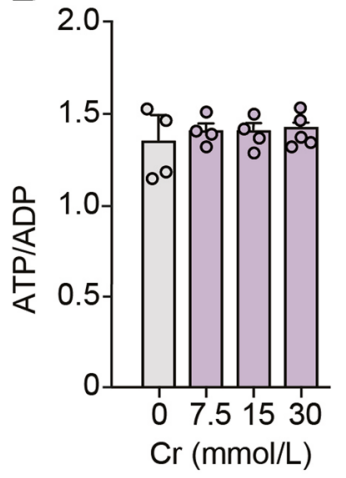

C

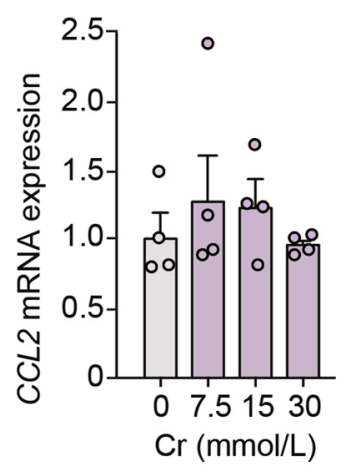

$\mathrm{O}=$ Oligomycin $\mathrm{F}=\mathrm{FCCP} \quad \mathrm{R} / \mathrm{A}=$ Rotenone/Antimycin

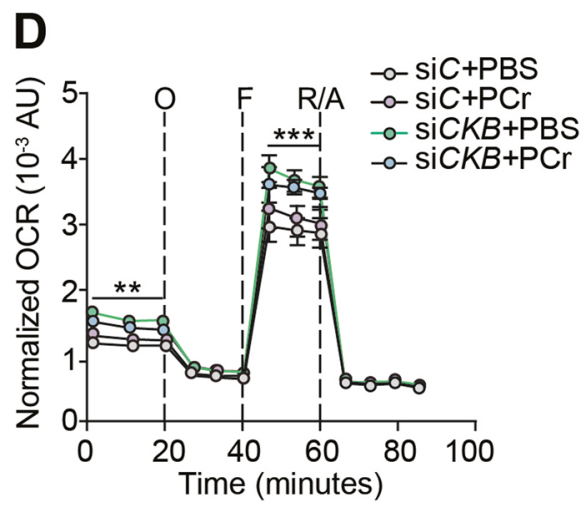

E

F
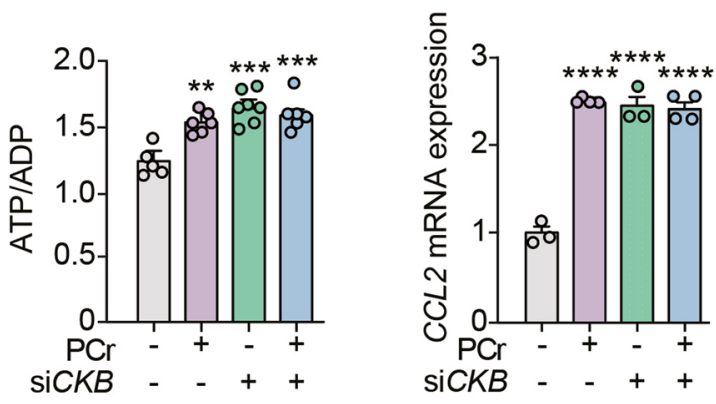

$\mathrm{O}=$ Oligomycin $\mathrm{F}=\mathrm{FCCP} \quad \mathrm{R} / \mathrm{A}=$ Rotenone/Antimycin

Extended Data Fig. 5 | Differential effects of creatine and phosphocreatine in human white adipocytes. a. Effect of creatine on OCR by Mito Stress test in in vitro human adipocytes transfected with either $\mathrm{siC}$ or $\mathrm{siCKB}$ incubated with or without creatine $(20 \mathrm{mmol} / \mathrm{L})$ (six replicates/condition) for $24 \mathrm{hours}$. Data are shown as mean \pm SEM. ${ }^{\star} p=0.029$ and ${ }^{\star \star} p=0.0012$ by one-way ANOVA. $\mathbf{b}$. ATP/ADP levels in in vitro differentiated human adipocytes incubated with creatine $(7.5-30 \mathrm{mmol} / \mathrm{L}$ ) for 24 hours (four replicates/condition). Data are shown as mean $\pm \mathrm{SEM}$. c. Same experimental setup as panel B but displaying CCL2 expression (four to five replicates/condition, experiment repeated twice). Data are shown as mean \pm SEM. d. In vitro differentiated human adipocytes transfected with either $\mathrm{SiC}$ or $\mathrm{SiCKB}$ were incubated with or without phosphocreatine $(30 \mathrm{mM})$ for 24 hours and the effects on OCR were determined by Mito Stress test (six replicates/condition, experiment repeated twice). Data are shown as mean \pm SEM. $p=0.007$ and 0.0004 for basal and maximal respiration by two-way ANOVA. e. ATP/ADP levels in in vitro differentiated human adipocytes transfected with either siC or siCKB, incubated with phosphocreatine $(30 \mathrm{mmol} / \mathrm{L})$ for 24 hours ( $\mathrm{siC}=$ five replicates, $\mathrm{si} C+\mathrm{PCr}=$ six replicates, siCKB= seven replicates, siCKB+PCr=six replicates). Data are shown as mean \pm SEM. Overall $p=0.0004$ by two-way ANOVA. Results from Tukey's post-hoc test are indicated by ${ }^{\star \star} p<0.01$, ${ }^{\star \star \star} p<0.001$, respectively. f. Same experimental setup as panel $\mathrm{E}$ but displaying $C C L 2$ expression ( $\mathrm{si} C=$ three replicates, $\mathrm{siC}+\mathrm{PCr}=$ four replicates, siCKB=three replicates, $\mathrm{si} C K B+\mathrm{PCr}=$ four replicates, experiment repeated twice). Data are shown as mean $\pm \mathrm{SEM}$. Overall $p<0.0001$ by two-way ANOVA. Results from Tukey's post-hoc test are indicated by ${ }^{\star \star \star \star} \mathrm{p}<0.001$. Abbreviations: $\mathrm{AU}=$ arbitrary unit, $\mathrm{Cr}=\mathrm{creatine}, \mathrm{OCR}=$ oxygen consumption rate, $\mathrm{PCr}=\mathrm{phosphocreatine}$. 
A

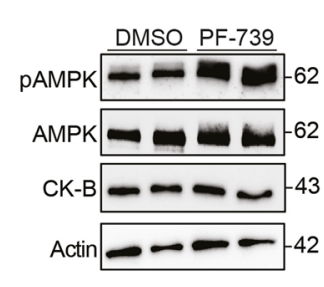

B

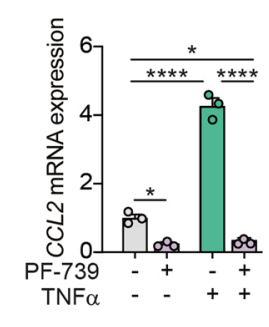

C

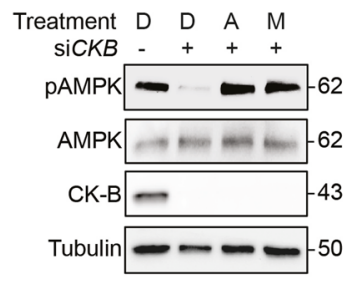

D

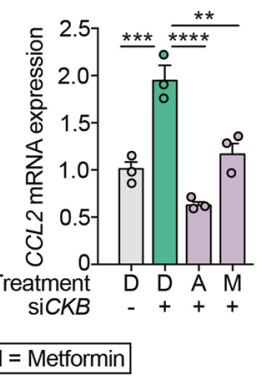

E

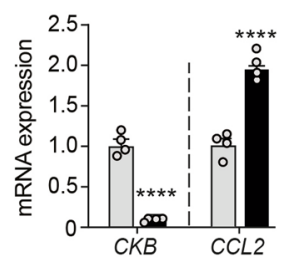

F

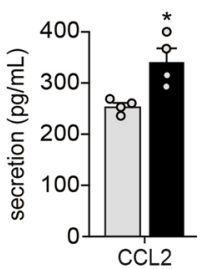

G

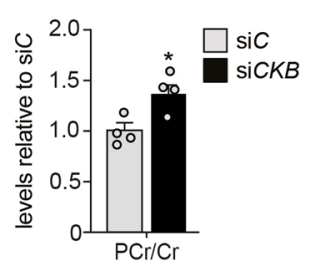

H

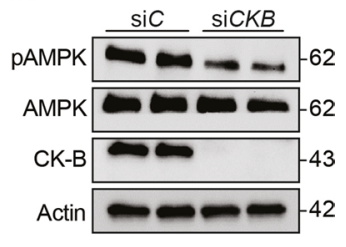

Extended Data Fig. 6 | AMPK regulates CCL2 expression. a. In vitro differentiated human adipocytes were incubated with DMSO or the AMPK activator PF-739 $(n=4)$. AMPK, phosphorylated AMPK (pAMPK), CK-B and actin levels were determined by western blot (in duplicates per blot). Left panel shows representative blots and bar graphs and to the right show quantifications of the indicated protein levels (four replicates/condition). Data are shown as mean \pm SEM. ${ }^{\star \star} p=0.0087$ by Student's two-sided t-test. b. CCL2 mRNA expression in in vitro differentiated human adipocytes following incubation with DMSO (control), TNF $\alpha$, PF-739 or both TNF $\alpha$ and PF-739 for 24 hours (three replicates/condition). Data are shown as mean \pm SEM. Overall $p<0.0001$ by two-way ANOVA. Results from Tukey's post-hoc test are indicated by ${ }^{\star} \mathrm{p}<0.05,{ }^{\star \star \star \star} \mathrm{p}<<0.0001$, respectively. c-d. AMPK, phosphorylated AMPK (pAMPK), CK-B and tubulin protein levels (C) as well as CCL2 expression (D) were determined in human in vitro differentiated adipocytes transfected with siC or siCKB, after incubation for 24 hours with DMSO or the AMPK activators AICAR and metformin (3 replicates/condition, experiment repeated twice). Data are shown as mean \pm SEM. Overall $p=0.0001$ by one-way ANOVA. Results from Tukey's post-hoc test are indicated by ${ }^{\star \star} p<0.01$, ${ }^{\star \star \star} p<0.001$, ${ }^{\star \star \star \star} p<0.0001$, respectively. e-f. CCL2 gene expression and secretion were determined in human in vitro differentiated adipocytes transfected with siC or siCKB (four replicates/condition) from a different vendor (Qiagen). Data are shown as mean \pm SEM. ${ }^{*} p=0.011,{ }^{\star \star * \star} p<0.0001$ by Student's two-sided t-test. g. Same experimental setup as panels E-F but displaying phosphocreatine/creatine levels. Data are shown as mean \pm SEM. ${ }^{*} p=0.012$ by Student's two-sided t-test. h. Same experimental setup as panels E-F but showing representative western blots of phosphorylated AMPK (pAMPK), AMPK, CK-B and actin (two replicates/condition loaded per blot). Abbreviations: $\mathrm{AICAR}=5$-aminoimidazole-4-carboxamide riboside, $\mathrm{AU}=\mathrm{arbitrary}$ unit, $\mathrm{Cr}=\mathrm{creatine}$, $\mathrm{PCr}=$ phosphocreatine. 
A
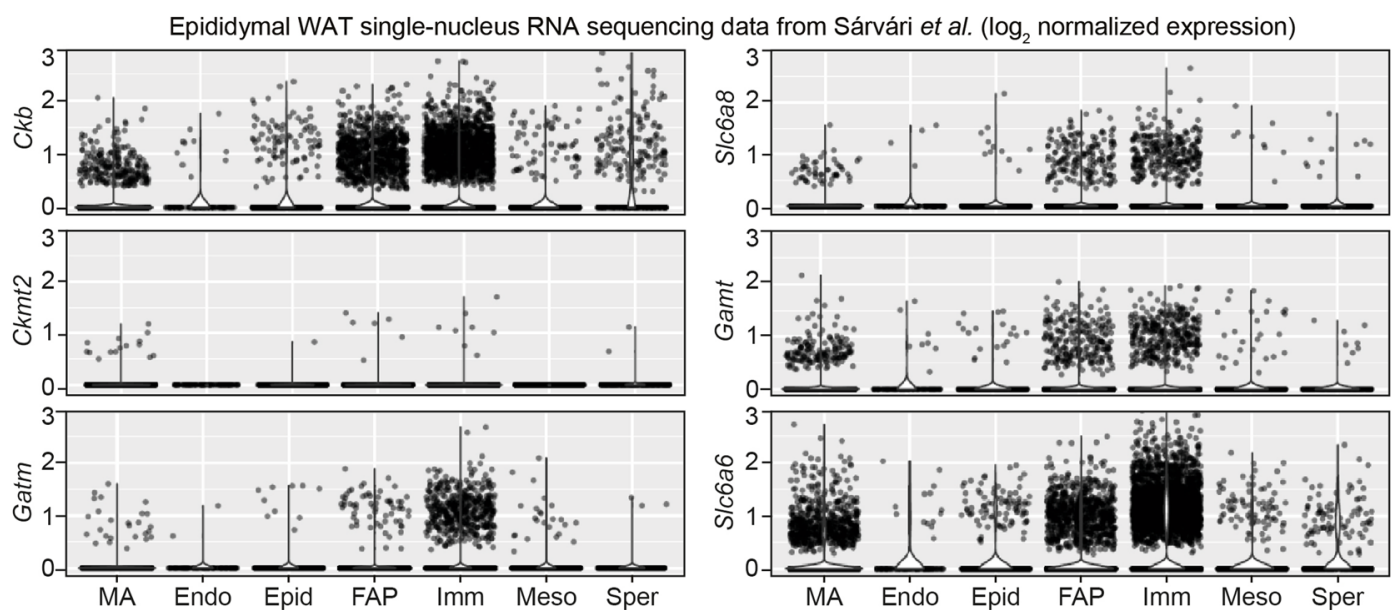

B

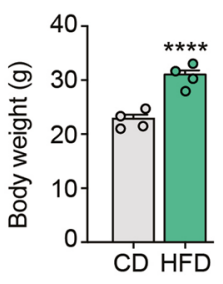

E

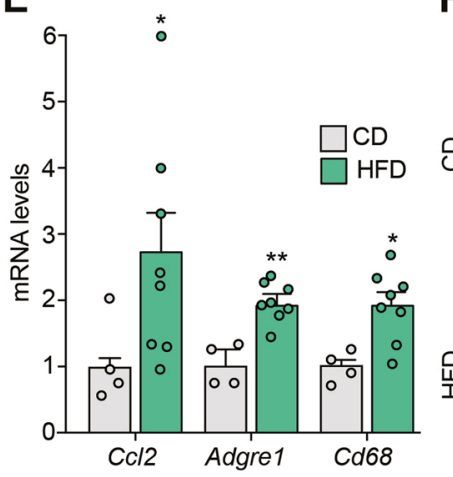

C

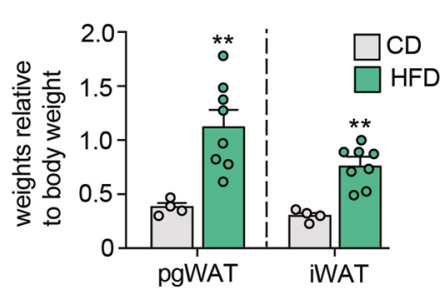

D

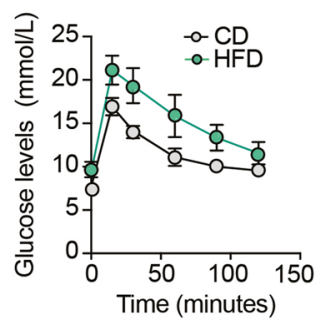

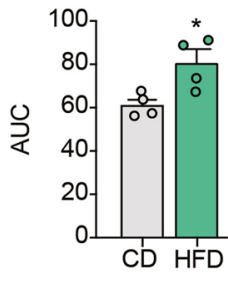

$\mathbf{F}$
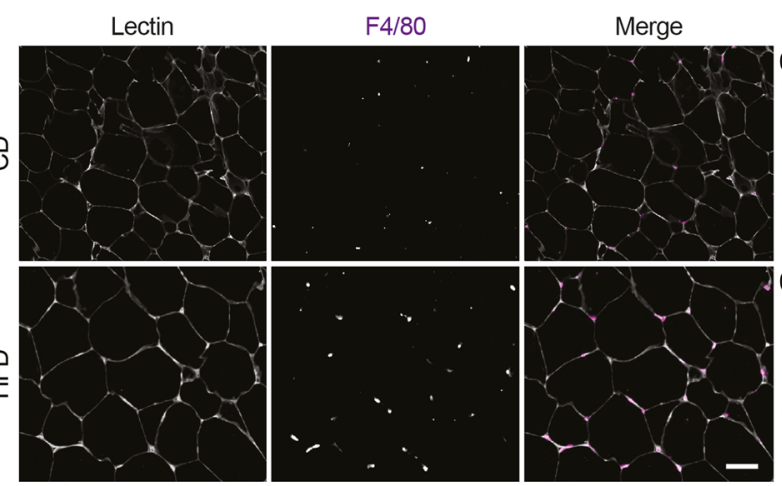

$\mathrm{F} 4 / 80^{+}$cells/field 020406080100

G H
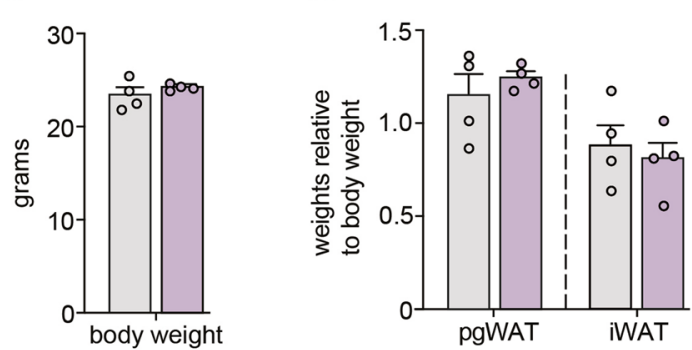

I
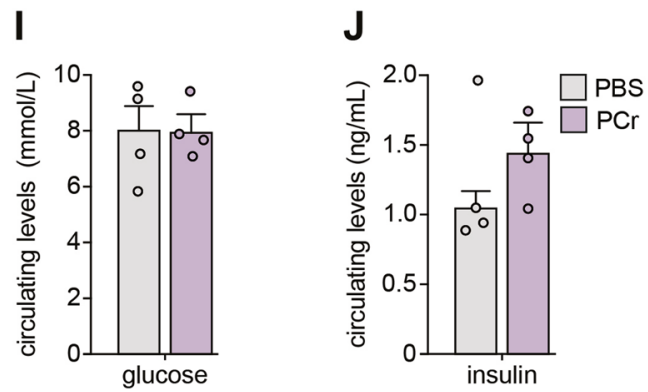

Extended Data Fig. 7 | See next page for caption. 
Extended Data Fig. 7 | Additional data from murine models. a. Expression of phosphocreatine/creatine-related genes in mouse WAT was extracted from single-nucleus RNA sequencing data published by Sárvári et $a^{\beta 34}$. b-c. Wild-type male mice were fed chow (CD) or high fat diet (HFD) for 5 weeks. Effects on body weight (four mice/condition) (B) and pgWAT and iWAT weights (four mice for CD and eight mice for HFD) (C) were determined. Data are shown as mean \pm SEM. ${ }^{\star \star} p=0.0034$ for pgWAT and 0.0016 for iWAT, respectively, ${ }^{\star \star \star \star} p<0.0001$ by Student's two-sided t-test. $\mathbf{d}$. Same experimental setup as in panels B-C but displaying effects on glucose tolerance by intraperitoneal glucose tolerance tests (four mice/group). The area under the curve (AUC) is displayed in the right panel. Data are shown as mean \pm SEM. ${ }^{*} p=0.041$ by Student's two-sided t-test. e. Same experimental setup as in panels $\mathrm{B}-\mathrm{C}$ but displaying effects on the expression of genes encoding pro-inflammatory markers/factors in pgWAT (four mice for CD and eight mice for HFD). Data are shown as mean \pm SEM. $p=0.049$ for $C c 12,0.002$ for Adgre1 and 0.023 for Cd68, respectively by Student's two-sided t-test. f. Representative immunofluorescence microphotographs of F4/80 (magenta) and Lens culinaris agglutinin (Lectin, gray) in pgWAT of HFD and CD mice. The number of $\mathrm{F} 4 / 80^{+}$cells was counted in 4 random fields $\left(n=4\right.$ mice/group). Scale bar $=50 \mu \mathrm{m}$. Data are shown as mean \pm SEM. ${ }^{\star *} p=0.0039$, by Student's two-sided t-test. g-j. Male mice on chow diet were injected intraperitoneally with PBS or phosphocreatine ( $3 \mathrm{mg} / \mathrm{g})$ for seven days $(n=4 \mathrm{mice} / \mathrm{group})$. Effects on body weight (G), pgWAT and iWAT weights $(\mathrm{H})$ and circulating glucose $(\mathrm{I})$ as well as insulin $(\mathrm{J})$ levels were determined. Data are shown as mean $\pm \mathrm{SEM}$. Abbreviations: $A \cup C=$ area under the curve, $C D=$ chow diet, Endo=endothelial, Epid=Edipidymal, $F A P=$ fibro-adipogenic progenitor, $\mathrm{HFD}=$ high fat diet, Imm=immune, iWAT=inguinal WAT, MA = mature adipocytes, Meso=mesothelial, $\mathrm{PCr}=$ phosphocreatine, pgWAT=perigonadal WAT, Sper=spermatozoa. 


\section{nature portfolio}

Corresponding author(s): Mejhert and Rydén NATMETAB-A21044659B

Last updated by author(s): Dec 23, 2021

\section{Reporting Summary}

Nature Portfolio wishes to improve the reproducibility of the work that we publish. This form provides structure for consistency and transparency in reporting. For further information on Nature Portfolio policies, see our Editorial Policies and the Editorial Policy Checklist.

\section{Statistics}

For all statistical analyses, confirm that the following items are present in the figure legend, table legend, main text, or Methods section.

n/a Confirmed

\The exact sample size $(n)$ for each experimental group/condition, given as a discrete number and unit of measurement

$\square$ A statement on whether measurements were taken from distinct samples or whether the same sample was measured repeatedly

$\square$ The statistical test(s) used AND whether they are one- or two-sided

Only common tests should be described solely by name; describe more complex techniques in the Methods section.

$\bigotimes$ A description of all covariates tested

$\square \bigotimes$ A description of any assumptions or corrections, such as tests of normality and adjustment for multiple comparisons

$\triangle$ A full description of the statistical parameters including central tendency (e.g. means) or other basic estimates (e.g. regression coefficient)

$\bigotimes$ AND variation (e.g. standard deviation) or associated estimates of uncertainty (e.g. confidence intervals)

For null hypothesis testing, the test statistic (e.g. $F, t, r$ ) with confidence intervals, effect sizes, degrees of freedom and $P$ value noted

Give $P$ values as exact values whenever suitable.

Х $\square$ For Bayesian analysis, information on the choice of priors and Markov chain Monte Carlo settings

Х $\square$ For hierarchical and complex designs, identification of the appropriate level for tests and full reporting of outcomes

Х $\square$ Estimates of effect sizes (e.g. Cohen's $d$, Pearson's $r$ ), indicating how they were calculated

Our web collection on statistics for biologists contains articles on many of the points above.

\section{Software and code}

Policy information about availability of computer code

Data collection AxioVision 4.9.1, NIS-Elements.

Data analysis MassHunter, Excel 365, GraphPad Prism 7, JMP v15.1, SPSS v24, ImageJ 1.45 and R v4.1.1

For manuscripts utilizing custom algorithms or software that are central to the research but not yet described in published literature, software must be made available to editors and reviewers. We strongly encourage code deposition in a community repository (e.g. GitHub). See the Nature Portfolio guidelines for submitting code \& software for further information.

\section{Data}

Policy information about availability of data

All manuscripts must include a data availability statement. This statement should provide the following information, where applicable:

- Accession codes, unique identifiers, or web links for publicly available datasets

- A description of any restrictions on data availability

- For clinical datasets or third party data, please ensure that the statement adheres to our policy

Microarray data generated in this study are publicly available in the NCBI Gene Expression Omnibus repository under the accession number GSE192361. Additional data used in this work are available using GEO numbers detailed in the paper under the section Data Availability. 


\section{Field-specific reporting}

Please select the one below that is the best fit for your research. If you are not sure, read the appropriate sections before making your selection.

$\bigotimes$ Life sciences $\quad \square$ Behavioural \& social sciences $\quad \square$ Ecological, evolutionary \& environmental sciences

For a reference copy of the document with all sections, see nature.com/documents/nr-reporting-summary-flat.pdf

\section{Life sciences study design}

All studies must disclose on these points even when the disclosure is negative.

Sample size Human omics data were retrospectively analyzed from previously described independent patient cohorts (see references in the Results section). For animal experiments, the number of mice was based on previous experience. No sample size calculation was therefore performed.

Data exclusions No data were excluded

Replication Experimental findings were repeated multiple times in independent experiments as indicated in the figure legends

Randomization Experiments in wild-type and knockout animals were not randomized as mice were identified by genotyping. In other experiments, samples/ mice treated with siRNA/drugs/diets, were randomly assigned to each group.

Blinding $\quad$ For the mouse studies, the investigators were not blinded as they handled the phenotyping and sacrification of the mice. However, the investigators were blinded for sample processing and samples were processed in random order.

\section{Reporting for specific materials, systems and methods}

We require information from authors about some types of materials, experimental systems and methods used in many studies. Here, indicate whether each material, system or method listed is relevant to your study. If you are not sure if a list item applies to your research, read the appropriate section before selecting a response.

\begin{tabular}{l|l} 
Materials \& experimental system \\
\hline n/a & Involved in the study \\
$\square$ & $\bigotimes$ Antibodies \\
$\square$ Eukaryotic cell lines \\
$\square$ Palaeontology and archaeology \\
$\square$ Animals and other organisms \\
$\square$ & $\square$ Clinical data \\
$\square$ Dual use research of concern
\end{tabular}

\begin{tabular}{l|l}
\multicolumn{2}{l}{ Methods } \\
\hline n/a & Involved in the study \\
\hline & $\square$ ChIP-seq \\
$\searrow$ & $\square$ Flow cytometry \\
$\triangle$ & $\square$ MRI-based neuroimaging
\end{tabular}

\section{Antibodies}

Antibodies used

All antibodies used in this study are detailed in Supplementary Table 2 including catalogue numbers and manufacturers.

Validation

All antibodies used in this study were commercially available and validation data can be found on the respective manufacturers' websites. Key findings were validated by siRNA knockdown experiments.

\section{Animals and other organisms}

Policy information about studies involving animals; ARRIVE guidelines recommended for reporting animal research

Laboratory animals

For Ckb floxed animals, age and housing conditions are detailed in Rahbani et al (PMID 33597756).

For HFD and phosphocreatine injection studies, C57BL/6J male mice were used and were sacrificed at 10-12 weeks of age. Animals were housed at the KM-B animal facility at the Karolinska Institutet in ventilated cages ( 4 animals/cage) with a 12 hour light/12 hour dark cycle (lights on 06:00-18:00) in a temperature-controlled (20-24 ${ }^{\circ} \mathrm{C}, 50 \%$ humidity) facility with ad libitum access to food and water. Animals were handled following the European Union laws and guidelines for animal care. Health inventories were performed on a regular basis (every 3 months) and follow the guidelines of the Federation of European Laboratory Animal Science Associations.

Wild animals

No wild animals were included in the study.

Field-collected samples

No field-collected samples were used in this study. 


\section{Human research participants}

Policy information about studies involving human research participants

Population characteristics

Clinical characteristics of the four cohorts are detailed in Supplementary Table 1.

Recruitment

No new patients/research subjects were recruited for this study.

Ethics oversight

All studies based on existing human data sets were approved by the regional ethics board of the Stockholm County Council. Informed written consent was obtained from all participants. A compensation of approximately 100 EUR was given to participants undergoing fine needle biopsies according to Swedish guidelines and the approved ethical permits.

Note that full information on the approval of the study protocol must also be provided in the manuscript.

\section{Clinical data}

Policy information about clinical studies

All manuscripts should comply with the ICMJE guidelines for publication of clinical research and a completed CONSORT checklist must be included with all submissions.

Clinical trial registration

Study protocol

Data collection

Outcomes
NCT01727245 (cohort 1 and 4) and NCT01785134 (cohort 3)

The intervention (bariatric surgery) is described at clinicaltrials.gov

All subjects were investigated in the morning after an overnight fast at the Karolinska University Hospital, Huddinge. Subcutaneous white adipose tissue biopsies from the periumbilical area were obtained by needle biopsies. One part of the tissue was rinsed and snap frozen in liquid nitrogen and stored at $-80^{\circ} \mathrm{C}$ until analysis. One part was rinsed and fixed in PFA for immunohistochemistry. Details on additional analyses are provided in the paper including relevant references.

Primary and secondary outcome measures for each study are detailed at clinicaltrials.gov. 